MATHEMATICS OF COMPUTATION

Volume 77, Number 264, October 2008, Pages 2061-2084

S 0025-5718(08)02098-X

Article electronically published on May 29, 2008

\title{
NEW EXPANSIONS OF NUMERICAL EIGENVALUES FOR $-\Delta u=\lambda \rho u$ BY NONCONFORMING ELEMENTS
}

\author{
QUN LIN, HUNG-TSAI HUANG, AND ZI-CAI LI
}

\begin{abstract}
The paper explores new expansions of the eigenvalues for $-\Delta u=$ $\lambda \rho u$ in $S$ with Dirichlet boundary conditions by the bilinear element (denoted $Q_{1}$ ) and three nonconforming elements, the rotated bilinear element (denoted $Q_{1}^{\text {rot}}$ ), the extension of $Q_{1}^{\text {rot }}$ (denoted $E Q_{1}^{r o t}$ ) and Wilson's elements. The expansions indicate that $Q_{1}$ and $Q_{1}^{r o t}$ provide upper bounds of the eigenvalues, and that $E Q_{1}^{r o t}$ and Wilson's elements provide lower bounds of the eigenvalues. By extrapolation, the $O\left(h^{4}\right)$ convergence rate can be obtained, where $h$ is the maximal boundary length of uniform rectangles. Numerical experiments are carried out to verify the theoretical analysis made.
\end{abstract}

\section{INTRODUCTION}

In this paper, we consider the eigenvalue problem

$$
\begin{array}{cl}
-\Delta u=\lambda \rho u & \text { in } S, \\
u=0 & \text { in } \partial S,
\end{array}
$$

where $S=[0,1]^{2}$, the function $\rho=\rho(x, y)>0$ and $\rho \in C^{2}(S)$. Then Eqs. (1.1) and (1.2) can be written in a weak form: To seek $(\lambda, u) \in R \times H_{0}^{1}(S)$ with $u \neq 0$ such that

$$
a(u, v)=\lambda(u, v), \forall v \in H_{0}^{1}(S),
$$

where $H_{0}^{1}(S)=\left\{v\left|v \in H^{1}(S), v\right|_{\partial S}=0\right\}$, and

$$
\begin{aligned}
& a(u, v)=\iint_{S} \nabla u \nabla v, \\
& (u, v)=\iint_{S} \rho u v .
\end{aligned}
$$

We choose one conforming element, the bilinear element $Q_{1}$, and three nonconforming elements: the rotated $Q_{1}$ (denoted $Q_{1}^{\text {rot }}$ ), the extension of $Q_{1}^{\text {rot }}$ (denoted $E Q_{1}^{r o t}$ ) and Wilson's element. All the above elements are defined on rectangles $\square_{i j}$ (see Figure 1), and their admissible functions are defined as follows.

(1) Bilinear element $Q_{1}$. The piecewise interpolation functions $u_{I} \in Q_{1}=$ $\operatorname{span}\{1, x, y, x y\}$ are formulated as

$$
u\left(Z_{i}\right)=u_{I}\left(Z_{i}\right), i=1,2,3,4,
$$

Received by the editor February 24, 2006 and, in revised form, February 14, 2007.

2000 Mathematics Subject Classification. Primary 65N30.

Key words and phrases. Bilinear elements, rotated bilinear element, the extension of rotated bilinear element, Wilson's element, eigenvalue problem, extrapolation, global superconvergence.

(C)2008 American Mathematical Society Reverts to public domain 28 years from publication 

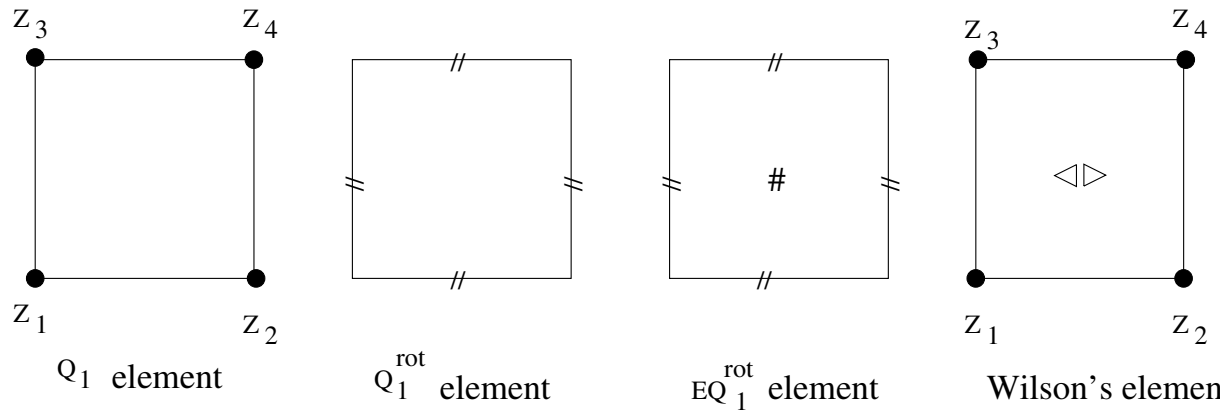

Wilson's element

Figure 1. The rectangular elements, where // and \# denote the line and the area elements, respectively, and $\triangleleft$ and $\triangleright$ denote $u_{x x}$ and $u_{y y}$ at the center, respectively.

where $Z_{i}$ are the four corners of $\square_{i j}$, and $\square_{i j}=\left\{(x, y) \mid x_{i}-h_{i} \leq x \leq\right.$ $\left.x_{i}+h_{i}, y_{j}-k_{j} \leq y \leq y_{j}+k_{j}\right\}$.

(2) Rotated $Q_{1}$ element $\left(Q_{1}^{r o t}\right)$. The piecewise interpolation functions $u_{I} \in$ $\operatorname{span}\left\{1, x, y, x^{2}-y^{2}\right\}$ are formulated by

$$
\int_{\ell_{k}} u=\int_{\ell_{k}} u_{I}, k=1,2,3,4,
$$

where $\ell_{k}$ are the edges of $\square_{i j}$.

(3) Extension of $Q_{1}^{\text {rot }}\left(E Q_{1}^{r o t}\right)$. The piecewise interpolation functions $u_{I} \in$ $\operatorname{span}\left\{1, x, y, x^{2}, y^{2}\right\}$ are formulated by

$$
\begin{aligned}
& \int_{\ell_{k}} u=\int_{\ell_{k}} u_{I}, k=1,2,3,4, \\
& \iint_{\square_{i j}} u=\iint_{\square_{i j}} u_{I} .
\end{aligned}
$$

(4) Wilson's element. The piecewise interpolation functions $u_{I} \in P_{2}=$ $\operatorname{span}\left\{1, x, y, x y, x^{2}, y^{2}\right\}$ are formulated by

$$
\begin{aligned}
& u\left(Z_{i}\right)=u_{I}\left(Z_{i}\right), i=1,2,3,4, \\
& u_{x x}(O)=\left(u_{I}\right)_{x x}(O), u_{y y}(O)=\left(u_{I}\right)_{y y}(O),
\end{aligned}
$$

where $O$ is the center of $\square_{i j}$.

Let $S=\bigcup_{i j} \square_{i j}$, where $\square_{i j}$ are quasi-uniform. Denote by $V_{h}^{0} \in L^{2}(S)$ the finite-dimensional collection of the admissible functions defined in $Q_{1}, Q_{1}^{\text {rot }}, E Q_{1}^{\text {rot }}$ and Wilson's elements. The conforming $Q_{1}$ element is used to seek the solution $\left(\lambda_{h}, u_{h}\right) \in R \times V_{h}^{0}\left(V_{h}^{0} \subset H_{0}^{1}(S)\right)$ such that

$$
a\left(u_{h}, v\right)=\lambda_{h}\left(u_{h}, v\right), \forall v \in V_{h}^{0},
$$

and the nonconforming elements, such as $Q_{1}^{\text {rot }}, E Q_{1}^{\text {rot }}$ and Wilson's elements, are used to seek $\left(\lambda_{h}, u_{h}\right) \in R \times V_{h}^{0}$ 1 such that

$$
a_{h}\left(u_{h}, v\right)=\lambda_{h}\left(u_{h}, v\right), \forall v \in V_{h}^{0},
$$

\footnotetext{
${ }^{1}$ Here $V_{h}^{0}$ is not a subset of $H_{0}^{1}(S)$.
} 
where

$$
a_{h}(u, v)=\sum_{i j} \iint_{\square_{i j}} \nabla u \nabla v .
$$

In $Q_{1}$ and Wilson's elements, the nodal variables are used, but in $Q_{1}^{\text {rot }}$ and $E Q_{1}^{r o t}$, the line and the area variables are also chosen, which can be interpreted as the average values on the edges $\partial \square_{i j}$ and those in the area $\square_{i j}$. The line-area interpolation in $Q_{1}^{r o t}$ and $E Q_{1}^{\text {rot }}$ is, rather than the nodal interpolation, advantageous in global superconvergence.

In this paper, we explore the expansions of the eigenvalues $\lambda_{h}$. When $\square_{i j}$ are uniform squares with the boundary length $h$, we obtain the following formulas:

$$
\lambda_{h}-\lambda=\left\{\begin{array}{lc}
\frac{h^{2}}{3} \iint_{S}\left(u_{x x}^{2}+u_{y y}^{2}\right)+O\left(h^{4}\right), & \text { for } Q_{1} \text { element, } \\
\frac{h^{2}}{6} \iint_{S}\left(u_{x x}-u_{y y}\right)^{2}+O\left(h^{4}\right), & \text { for } Q_{1}^{\text {rot }} \text { element } \\
-\frac{2 h^{2}}{3} \iint_{S} u_{x y}^{2}+O\left(h^{4}\right), & \text { for } E Q_{1}^{r o t} \text { element } \\
-\frac{2 h^{2}}{3} \iint_{S} u_{x x} u_{y y}-\frac{h^{2}}{3} \iint_{S}\left[u_{x x}\left(u_{h}\right)_{y y}+u_{y y}\left(u_{h}\right)_{x x}\right]+O\left(h^{4}\right), & \text { for Wilson's element. }
\end{array}\right.
$$

The detailed proof for $Q_{1}^{\text {rot }}$ and $E Q_{1}^{\text {rot }}$ elements is deferred to Section 3, and the proof for $Q_{1}$ and Wilson's elements will appear elsewhere. From the expansions of $\lambda_{h}$ in (1.15), we may draw a few important conclusions:

(1) Both $Q_{1}$ and $Q_{1}^{r o t}$ provide an upper bound of $\lambda$, but in contrast, $E Q_{1}^{\text {rot }}$ and Wilson's elements provide a lower bound of $\lambda$. The lower estimation of $\lambda$ is particularly interesting, because all conforming FEMs can only provide an upper estimation on $\lambda$.

(2) Suppose that $\rho(x, y)$ is symmetric with respect to $x$ and $y$. For the minimal eigenvalue $\lambda_{\min }=\lambda_{1}$, since the corresponding eigenfunction satisfies $u_{x x}=$ $u_{y y}$, the $Q_{1}^{\text {rot }}$ element yields the high $O\left(h^{4}\right)$ convergence rate. Such an ultraconvergence of $Q_{1}^{r o t}$ is retained for any eigenvalue whose corresponding eigenfunction is symmetric with respect to $x$ and $y$.

(3) The errors of $\lambda$ by $Q_{1}, Q_{1}^{r o t}$ and $E Q_{1}^{r o t}$ have the following relation:

$$
\left.E\right|_{Q_{1}^{\text {rot }}}-\frac{1}{2}\left(\left.E\right|_{Q_{1}}+\left.E\right|_{E Q_{1}^{r o t}}\right)=O\left(h^{4}\right)
$$

where $E=\lambda_{h}-\lambda$.

(4) By the extrapolation we may reach the high $O\left(h^{4}\right)$ convergence rates for $Q_{1}, Q_{1}^{r o t}, E Q_{1}^{r o t}$, and Wilson's elements.

In our numerical experiments, the $O\left(h^{4}\right)$ convergence rate has been confirmed by the extrapolation for all four elements, and the further extrapolation can be carried out for the $Q_{1}$ element to reach the $O\left(h^{2 k}\right)(k \geq 2)$ convergence rates.

Let us mention the references related to this paper. Numerical eigenvalues are discussed in Babuska and Osborn [1, 2, 3], Chatelin [6], Koluta 10, Mercier et al. 
[17, Pierce and Varga [18, Rannacher [19, Strang and Fix [20, Wu [22] and Yang [23, 24]. The nonconforming elements, such as the rotated bilinear element (i.e., $\left.Q_{1}^{r o t}\right)$ and Wilson's element, are studied in Chen and Li [7, Hu et al. 9, Lua and Lin [16], and Lin and Lin [13], and the extrapolations for eigenvalues are explored in Blum et al. 4, Lin [12, Lin and Zhu [14, and Lü et al. [15.

It is worth pointing out that asymptotic lower bounds for eigenvalues have been obtained by the finite difference method (FDM) in Forsythe 8 and Weinberger 21 . In [8], for a convex $S$, the numerical eigenvalues by the standard five-node finite difference equations have lower bounds, and upper and lower bounds of numerical eigenvalues by FDM are also discussed in 21]. Since the FDM can be regarded as a special kind of FEM involving different integration rules in Li [11, the variational crimes, the terminology used in 20. for FEM with nonconforming elements and numerical integration, may produce the lower bounds of approximate eigenvalues.

\section{BASIC THEOREMS}

We rewrite (1.3) as:

$$
a(u, v)=(f, v), \forall v \in H_{0}^{1}(S),
$$

where $f=\lambda u$. Define the finite element projection $R_{h}$ by

$$
a_{h}\left(R_{h} u, v\right)=(f, v), \forall v \in V_{h}^{0} .
$$

For simplicity, we assume the simple eigenvalues, and consider only a few leading eigenvalues

$$
\lambda_{1}<\lambda_{2} \leq \ldots \leq \lambda_{k},
$$

where $k$ is a small integer. Note that the minimal eigenvalue $\lambda_{1}=\lambda_{\min }$ is of great interest in practical applications.

For the above elements, we cite the known results in [23, 24] as a lemma.

Lemma 2.1. For the quasi-uniform $\square_{i j}$ with the maximal boundary length $h$, there exists the follwing bound for leading eigenvalues $\lambda$ and their corresponding eigenfunctions $u$ :

$$
\left|\lambda-\lambda_{h}\right|+\left\|u-u_{h}\right\|_{0, S}+\left\|u-R_{h} u\right\|_{0, S} \leq C h^{2},
$$

where $C$ is a constant independent of $h$, and $\left(\lambda_{h}, u_{h}\right)$ are the FEM solutions by $Q_{1}$, $Q_{1}^{\text {rot }}, E Q_{1}^{\text {rot }}$ and Wilson's elements.

Below we give a new theorem.

Theorem 2.1 (Nonconforming). Let $\square_{i j}$ be quasi-uniform with the maximal boundary length $h$. For the nonconforming elements, there exists the error formula

$$
\lambda_{h}-\lambda=\lambda\left(u-u_{I}, u_{h}\right)-a_{h}\left(u-u_{I}, u_{h}\right)+a_{h}\left(u-R_{h} u, u_{h}\right)+O\left(h^{4}\right),
$$

where $u$ and $u_{I}$ are the true solution (i.e., eigenfunction) and the FEM interpolation of $u$, respectively, and $u_{h}$ and $R_{h} u$ are the FEM solution of (1.13) and the FEM projection in (2.2), respectively.

Proof. For the eigenfunctions,

$$
(u, u)=1,\left(u_{h}, u_{h}\right)=1 .
$$


We choose a different scale of $u_{h}$ by $\bar{u}_{h}=\frac{u_{h}}{\left(u, u_{h}\right)}$. Then we have $\left(u, \bar{u}_{h}\right)=1$, which yields

$$
\lambda_{h}=\lambda_{h}\left(u, \bar{u}_{h}\right)=\lambda_{h}\left(R_{h} u, \bar{u}_{h}\right)+\lambda_{h}\left(u-R_{h} u, \bar{u}_{h}\right) .
$$

Moreover, from (1.13) and (2.2), we obtain

$$
\lambda_{h}\left(R_{h} u, \bar{u}_{h}\right)=a_{h}\left(R_{h} u, \bar{u}_{h}\right)=\lambda\left(u, \bar{u}_{h}\right)=\lambda .
$$

Since $\bar{u}_{h}$ has a small difference from $u_{h}$, we obtain from Lemma 2.1.

$$
\left\|\bar{u}_{h}-u_{h}\right\|_{0, S}=\left\|\frac{\left(u, u-u_{h}\right) u_{h}}{\left(u, u_{h}\right)}\right\|_{0, S} \leq C h^{2} .
$$

Hence by means of Lemma 2.1 again, a primary expansion from (2.7) $-(2.9)$ is given by

$$
\lambda_{h}=\lambda+\lambda_{h}\left(u-R_{h} u, \bar{u}_{h}\right)=\lambda+\lambda_{h}\left(u-R_{h} u, u_{h}\right)+O\left(h^{4}\right) .
$$

Finally, a further expansion can be obtained:

$$
\begin{aligned}
\lambda_{h} & =\lambda+\lambda_{h}\left(u-u_{I}, u_{h}\right)+\lambda_{h}\left(u_{I}-R_{h} u, u_{h}\right)+O\left(h^{4}\right) \\
& =\lambda+\lambda_{h}\left(u-u_{I}, u_{h}\right)+a_{h}\left(u_{I}-R_{h} u, u_{h}\right)+O\left(h^{4}\right) \\
& =\lambda+\lambda\left(u-u_{I}, u_{h}\right)+a_{h}\left(u_{I}-u, u_{h}\right)+a_{h}\left(u-R_{h} u, u_{h}\right)+O\left(h^{4}\right),
\end{aligned}
$$

where we have replaced $\lambda_{h}$ by $\lambda$ from Lemma 2.1. This is the desired result (2.5), and completes the proof of Theorem 2.1 .

In Theorem 2.1, in order to derive the errors $\lambda_{h}-\lambda$, we need to evaluate the following interpolation errors:

$$
\left(u-u_{I}, v\right), a_{h}\left(u-u_{I}, v\right), \forall v \in V_{h}^{0},
$$

and the projection error

$$
a_{h}\left(u-R_{h} u, v\right), \forall v \in V_{h}^{0} .
$$

Note that the projection error (2.13) is null for the conforming element:2 and that the estimation of (2.12) is similar to that for Poisson's equation. Hence the key analysis of the nonconforming elements is to derive the expansions of (2.13). In this paper, the detailed proof is provided only for $Q_{1}^{r o t}$ and $E Q_{1}^{r o t}$ (see the next section), and the proof for the $Q_{1}$ and Wilson's elements in (1.15) appears elsewhere.

In error estimates, we often use the Bramble-Hilbert lemma [5: Denote by $B(u)$ a bounded linear function from $H^{k}(S)$ to $R 3$ If for a polynomial $P_{k}$ of degree $k$, $B\left(P_{k}\right)=0$, then there exists a constant $C$ independent of $u$ such that

$$
|B(u)| \leq C|u|_{k+1, S} .
$$

In this paper, we need more expansions of higher terms of degree $k+1$. We solicit the generalized Bramble-Hilbert Lemma. Let

$$
B(u)=\sum_{|\alpha|=k+1} \frac{B\left(x^{\alpha}\right)}{\alpha !|S|} \iint_{S} D^{\alpha} u+H(u)
$$

\footnotetext{
${ }^{2}$ For the conforming $Q_{1}$ element, the expansions of (2.12) will lead to those in (1.15), by using the same proof techniques in this paper.

${ }^{3}$ The bounded linear function $B(u)$ implies that it is continuous.
} 
where $x^{\alpha}=x_{1}^{\alpha_{1}} x_{2}^{\alpha_{2}}, \alpha_{1}+\alpha_{2}=\alpha$, and $\alpha !=\alpha_{1} ! \alpha_{2}$ !. H(u) in 2.15) is also a bounded linear function from $H^{k+1}(S)$ to $R$. We write the following lemma without proof, whose proof is given in Lin and Lin [13].

Lemma 2.2 (Generalized Bramble and Hilbert Lemma). Let $u \in H^{k+2}(S)$ and $B\left(P_{k}\right)=0$. Suppose that $H\left(P_{k+1}\right)=0$ in (2.15). There exists a bound,

$$
|H(u)| \leq C|u|_{k+2, S},
$$

where $C$ is a constant independent of $u$.

\section{3. $Q_{1}^{\text {rot }}$ AND $E Q_{1}^{\text {rot }}$ ELEMENTS}

In this paper, we will derive the expansions in (1.15) for $Q_{1}^{\text {rot }}$ and $E Q_{1}^{\text {rot }}$. We merge their proofs together, because the main proof for both nonconforming elements has many features in common. Based on Theorem 2.1, the three terms in (2.5) need to be evaluated. For both $Q_{1}^{r o t}$ and $E Q_{1}^{r o t}$, from their definition of $u_{I}$ and by integration by parts, we can show the following equality easily:

$$
a_{h}\left(u-u_{I}, v\right)=\iint_{S} \nabla\left(u-u_{I}\right) \nabla v=0, \forall v \in Q_{1}^{r o t} \text { or } E Q_{1}^{r o t} .
$$

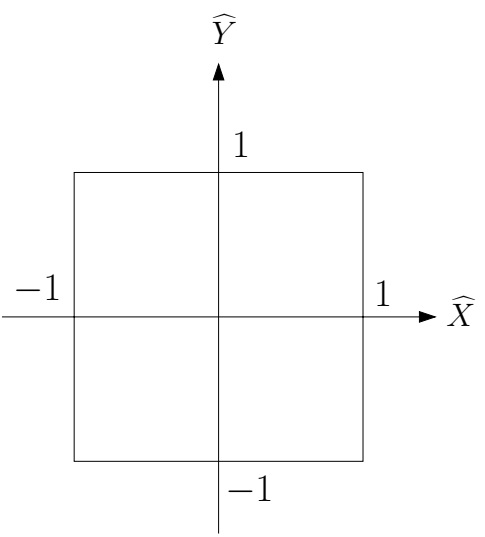

(1)

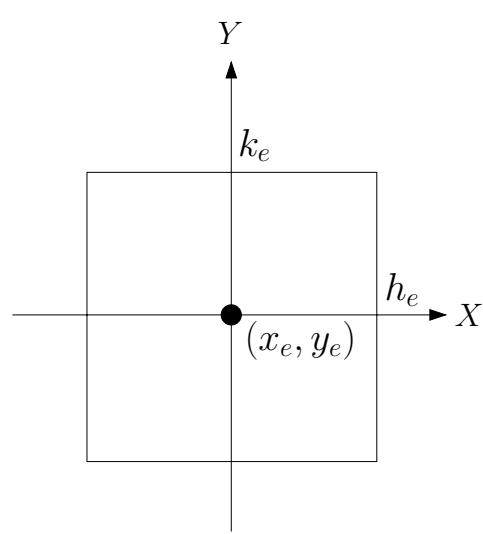

$(2)$

Figure 2. (1) $\widehat{e}=[-1,1] \times[-1,1] .(2) e=\square_{i j}=\left[x_{e}-h_{e}, x_{e}+\right.$ $\left.h_{e}\right] \times\left[y_{e}-k_{e}, y_{e}+k_{e}\right]$.

To obtain the expansions of the other two terms in (2.12) and (2.13), we need the following lemmas.

Lemma 3.1. For $v \in E Q_{1}^{\text {rot }}$ or $Q_{1}^{\text {rot }}$, there exists the equality

$$
\begin{aligned}
a_{h}\left(u-R_{h} u, v\right)= & \sum_{e}\left[\frac{k_{e}^{2}}{3} \iint_{e} u_{x x y} v_{y}-\frac{4 k_{e}^{4}}{45} \iint_{e} u_{x x y y} v_{y y}\right. \\
& \left.+\frac{h_{e}^{2}}{3} \iint_{e} u_{y y x} v_{x}-\frac{4 h_{e}^{4}}{45} \iint_{e} u_{y y x x} v_{x x}\right]+O\left(h^{5}\right)|u|_{5}|v|_{2, h},
\end{aligned}
$$

where $|v|_{m, h}=\sqrt{\sum_{e}|v|_{m, e}^{2}}(m=1,2)$, and $e=\square_{i j}=\left[x_{e}-h_{e}, x_{e}+h_{e}\right] \times\left[y_{e}-\right.$ $\left.k_{e}, y_{e}+k_{e}\right]$ (see Figure 21). Moreover, for uniform rectangles $\square_{i j}$ with $h_{e}=h$ and 
$k_{e}=k$, there exists the following equality for $v \in E Q_{1}^{\text {rot }}$ or $Q_{1}^{\text {rot }}$ :

$$
a_{h}\left(u-R_{h} u, v\right)=-\frac{h^{2}+k^{2}}{3} \iint_{S} u_{x x y y} v+O\left(h^{4}\right)\|u\|_{5}\|v\|_{1, h} .
$$

Lemma 3.2. For $v \in E Q_{1}^{\text {rot }}(e)$,

(3.4) $\iint_{e}\left(u-u_{I}\right) v=-\frac{h_{e}^{4}}{45} \iint_{e} u_{x x x} v_{x}-\frac{k_{e}^{4}}{45} \iint_{e} u_{y y y} v_{y}+O\left(h^{5}\right)|u|_{4, e}|v|_{1, e}$.

Lemma 3.3. For $v \in Q_{1}^{\text {rot }}(e)$,

$$
\begin{aligned}
\iint_{e}\left(u-u_{I}\right) v= & -\frac{h^{2}}{6} \iint_{e}\left(u_{x x}+u_{y y}\right) v+\frac{h^{4}}{30} \iint_{e}\left(u_{x x x} v_{x}+u_{y y y} v_{y}\right) \\
& +\frac{h^{4}}{18} \iint_{e}\left(u_{x x y} v_{y}+u_{y y x} v_{x}\right)+O\left(h^{5}\right)|u|_{4, e}|v|_{1, e} .
\end{aligned}
$$

Lemma 3.4. For $v \in E Q_{1}^{\text {rot }}(e)$ or $Q_{1}^{\text {rot }}(e)$, there exists the integral equality

$$
\begin{aligned}
& \iint_{e} u_{x x}\left(\left(y-y_{e}\right) v_{y}\left(x, y_{e}\right)+\left(\left(y-y_{e}\right)^{2}-\frac{k_{e}^{2}}{3}\right) v_{y y}\left(x, y_{e}\right)\right) \\
= & \frac{k_{e}^{2}}{3} \iint_{e} u_{x x y} v_{y}-\frac{4 k_{e}^{4}}{45} \iint_{e} u_{x x y y} v_{y y}+O\left(h^{5}\right)|u|_{5, e}|v|_{2, e} .
\end{aligned}
$$

The proof of Lemmas 3.1-3.4 is deferred to Sections 3.1-3.4. For $E Q_{1}^{\text {rot }}$, we have the following theorem.

Theorem 3.1. Let $\square_{i j}$ be quasi-uniform. For $E Q_{1}^{r o t}$, there exists the eigenvalue error

$$
\lambda_{h}-\lambda=\frac{1}{3} \sum_{e}\left[\iint_{e} k_{e}^{2} u_{x x y} u_{y}+h_{e}^{2} \iint_{e} u_{x y y} u_{x}\right]+O\left(h^{3}\right) .
$$

Moreover for uniform $\square_{i j}$,

$$
\lambda_{h}-\lambda=-\frac{h^{2}+k^{2}}{3} \iint_{S} u_{x y}^{2}+O\left(h^{4}\right) .
$$

Proof. From Lemma 3.2 ,

$$
\begin{aligned}
& \lambda\left(u-u_{I}, u_{h}\right)=\lambda \iint_{S}\left(u-u_{I}\right) \rho u_{h} \\
= & \lambda \sum_{e}\left[\iint_{e}\left(u-u_{I}\right)\left(\rho u_{h}\right)_{I}-\iint_{e}\left(u-u_{I}\right)\left(\rho u_{h}-\left(\rho u_{h}\right)_{I}\right)\right] \\
= & -\lambda \sum_{e}\left[\frac{h_{e}^{4}}{45} \iint_{e} u_{x x x}\left(\left(\rho u_{h}\right)_{I}\right)_{x}-\frac{k_{e}^{4}}{45} \iint_{e} u_{y y y}\left(\left(\rho u_{h}\right)_{I}\right)_{y}\right]+O\left(h^{4}\right)=O\left(h^{4}\right),
\end{aligned}
$$

where we have used

$$
\iint_{e}\left(u-u_{I}\right)\left(\rho u_{h}-\left(\rho u_{h}\right)_{I}\right)=\iint_{e}\left(u-u_{I}\right) u_{h}\left(\rho-\rho_{I}\right)=O\left(h^{4}\right) .
$$

Also from Lemma 3.1 ,

$$
\begin{aligned}
a_{h}\left(u-R_{h} u, u_{h}\right) & =\sum_{e} \frac{k_{e}^{2}}{3} \iint_{e} u_{x x y}\left(u_{h}\right)_{y}+\sum_{e} \frac{h_{e}^{2}}{3} \iint_{e} u_{x y y}\left(u_{h}\right)_{x}+O\left(h^{4}\right) \\
& =\sum_{e} \frac{k_{e}^{2}}{3} \iint_{e} u_{x x y} u_{y}+\sum_{e} \frac{h_{e}^{2}}{3} \iint_{e} u_{x y y} u_{x}+O\left(h^{3}\right) .
\end{aligned}
$$


Based on Theorem 2.1, combining (3.1), (3.9) and (3.11) yields the first desired result (3.7).

Next, we prove (3.8) for the uniform rectangles $\square_{i j}$. From Lemmas 3.1 and 2.1 and by integration by parts,

$$
\begin{aligned}
a_{h}\left(u-R_{h} u, u_{h}\right)= & \frac{h^{2}}{3} \sum_{e} \iint_{e} u_{x y y}\left(u_{h}\right)_{x} \\
& +\frac{k^{2}}{3} \sum_{e} \iint_{e} u_{x x y}\left(u_{h}\right)_{y}+O\left(h^{4}\right)|u|_{5}\left|u_{h}\right|_{2, h} \\
= & -\frac{h^{2}+k^{2}}{3} \sum_{e} \iint_{e} u_{x x y y}\left(u_{h}\right)+O\left(h^{4}\right) \\
= & -\frac{h^{2}+k^{2}}{3} \sum_{e} \iint_{e} u_{x x y y} u+O\left(h^{4}\right) \\
= & -\frac{h^{2}+k^{2}}{3} \iint_{S} u_{x y}^{2}+O\left(h^{4}\right),
\end{aligned}
$$

where we have used the integration by parts again,

$$
\iint_{S} u_{x x y y} u=-\iint_{S} u_{x y y} u_{x}=\iint_{S} u_{x y}^{2}
$$

and

$$
\begin{aligned}
\left|u_{h}\right|_{2, h} & \leq\left|u_{h}-u_{I}\right|_{2, h}+\left|u_{I}-u\right|_{2, h}+|u|_{2} \\
& \leq C h^{-1}\left|u_{h}-u_{I}\right|_{1, h}+C|u|_{2} \leq C|u|_{2} .
\end{aligned}
$$

Based on Theorem 2.1] combining (3.1), (3.9) and (3.12) yield the second desired result (3.8) (i.e., (1.15) for $E Q_{1}^{\text {rot }}$ with $k_{e}=h_{e}=h$ ). This completes the proof of Theorem 3.1 .

Below, for $Q_{1}^{\text {rot }}$, we have the following theorem.

Theorem 3.2. Let $\square_{i j}$ be uniform squares. For $Q_{1}^{\text {rot }}$ there exists the eigenvalue error

$$
\lambda_{h}-\lambda=\frac{h^{2}}{6} \iint_{S}\left(u_{x x}-u_{y y}\right)^{2}+O\left(h^{4}\right) .
$$

Proof. For $Q_{1}^{\text {rot }}$ on uniform square $\square_{i j}$ with $h=k$, we have from Lemmas 3.3 and 2.1.

$$
\begin{aligned}
\lambda\left(u-u_{I}, u_{h}\right) & =\lambda \iint_{S}\left(u-u_{I}\right)\left(\rho u_{h}\right)_{I}+O\left(h^{4}\right) \\
& =-\lambda \frac{h^{2}}{6} \iint_{S}\left(u_{x x}+u_{y y}\right)\left(\rho u_{h}\right)_{I}+O\left(h^{4}\right) \\
& =-\lambda \frac{h^{2}}{6} \iint_{S}\left(u_{x x}+u_{y y}\right) \rho u+O\left(h^{4}\right) \\
& =\frac{h^{2}}{6} \iint_{S}\left(u_{x x}+u_{y y}\right)^{2}+O\left(h^{4}\right),
\end{aligned}
$$

where we have used (1.1). From integration by parts, there exists the equalilty

$$
\iint_{S} u_{x x} u_{y y}=\iint_{S} u_{x y}^{2}
$$


$\ell_{2}$

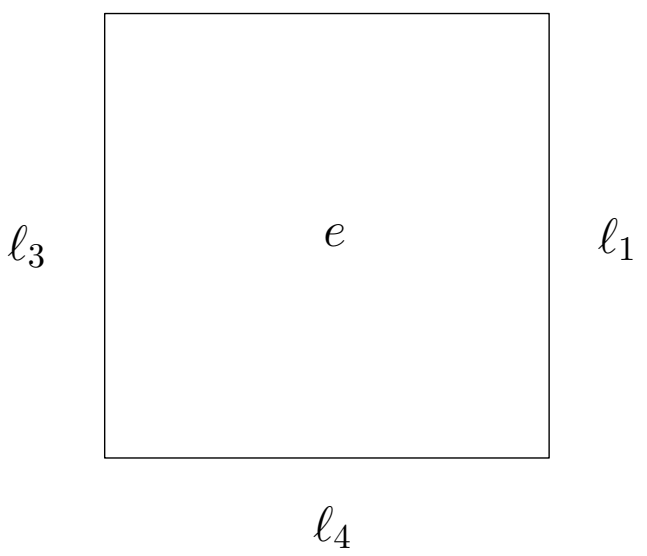

Figure 3. The rectangle.

For uniform squares $\square_{i j}$ with $h=k$, based on Theorem 2.1, combining (3.1), (3.12), (3.16) and (3.17) yields the desired result (3.15) (i.e., (1.15) for $Q_{1}^{\text {rot }}$ ). This completes the proof of Theorem 3.2

Theorems 3.1 and 3.2 provide the desired expansions in (1.15) for $E Q_{1}^{r o t}$ and $Q_{1}^{r o t}$ elements. It is interesting to note that $Q_{1}^{r o t}$ and $E Q_{1}^{r o t}$ give the upper and the lower bounds of the leading eigenvalues, respectively.

3.1. Proof of Lemma 3.1. For the nonconforming errors of $Q_{1}^{\text {rot }}$ and $E Q_{1}^{\text {rot }}$, we have from (2.1), (2.2) and the Green formula,

$$
\begin{aligned}
a_{h}\left(u-R_{h} u, v\right) & =\sum_{e} \oint_{\partial e} \frac{\partial u}{\partial n} v d s \\
& =\sum_{e}\left(\int_{\ell_{1}}-\int_{\ell_{3}}\right) u_{x} v d y+\sum_{e}\left(\int_{\ell_{2}}-\int_{\ell_{4}}\right) u_{y} v d x,
\end{aligned}
$$

where $\ell_{i}$ are the edges in Figure 3. Since the average on $\ell_{k}$ is continuous based on the definitions in (1.7) and (1.8), we have

$$
\sum_{e}\left(\int_{\ell_{1}}-\int_{\ell_{3}}\right) u_{x} \bar{v} d y=0
$$

where $\bar{v}=\int_{\ell_{i}} v d s /\left|\ell_{i}\right|$ is constant on $\ell_{i}$. Hence we obtain

$$
\sum_{e}\left(\int_{\ell_{1}}-\int_{\ell_{3}}\right) u_{x} v d y=\sum_{e}\left(\int_{\ell_{1}}-\int_{\ell_{3}}\right) u_{x}(v-\bar{v}) d y .
$$

Also since $\left.v\right|_{\ell_{1} \cup \ell_{3}}=\operatorname{span}\left\{1, y-y_{e},\left(y-y_{e}\right)^{2}\right\}$, then $\left.\bar{v}\right|_{\ell_{1} \cup \ell_{3}}=\operatorname{span}\left\{1,0, \frac{k_{e}^{2}}{3}\right\}$. Moreover, from Taylor's formula in the $y$ variable for each $x$, we have

$$
\left.(v-\bar{v})\right|_{\ell_{i}}=\left(y-y_{e}\right) v_{y}\left(x, y_{e}\right)+\left(\left(y-y_{e}\right)^{2}-\frac{k_{e}^{2}}{3}\right) \frac{v_{y y}\left(x, y_{e}\right)}{2}, i=1,3 .
$$


TABLE 1. The integration, $\iint_{\widehat{e}}\left(u-u_{I}\right) v$ for $u \in P_{3} \backslash E Q_{1}^{r o t}, v \in$ $E Q_{1}^{\text {rot }}=\operatorname{span}\left\{1, x, y, x^{2}, y^{2}\right\}$, where $\widehat{e}=[-1,1]^{2}$ and the sign $0^{+}$ denotes that the computed integrals are zero.

\begin{tabular}{|c||c||c|c|c|c||c|}
\hline$u$ & $x y$ & $x^{3}$ & $x^{2} y$ & $x y^{2}$ & $y^{3}$ & Notes \\
\hline$u_{I}$ & 0 & $x$ & $\frac{1}{3} y$ & $\frac{1}{3} x$ & $y$ & $/$ \\
\hline$u-u_{I}$ & $x y$ & $x^{3}-x$ & $x^{2} y-\frac{1}{3} y$ & $x y^{2}-\frac{1}{3} x$ & $y^{3}-y$ & $/$ \\
\hline \hline $\iint_{\widehat{e}}\left(u-u_{I}\right)$ & 0 & 0 & 0 & 0 & 0 & $v=1$ \\
\hline $\iint_{\widehat{e}}\left(u-u_{I}\right) x$ & 0 & $-\frac{8}{15}$ & 0 & $0^{+}$ & 0 & $v=x$ \\
\hline $\iint_{\widehat{e}}\left(u-u_{I}\right) y$ & 0 & 0 & $0^{+}$ & 0 & $-\frac{8}{15}$ & $v=y$ \\
\hline $\iint_{\widehat{e}}\left(u-u_{I}\right) x^{2}$ & 0 & 0 & 0 & 0 & 0 & $v=x^{2}$ \\
\hline $\iint_{\widehat{e}}\left(u-u_{I}\right) y^{2}$ & 0 & 0 & 0 & 0 & 0 & $v=y^{2}$ \\
\hline
\end{tabular}

Then

$$
\begin{aligned}
& \left(\int_{\ell_{1}}-\int_{\ell_{3}}\right) u_{x}(v-\bar{v}) d y \\
= & \left(\int_{\ell_{1}}-\int_{\ell_{3}}\right) u_{x}\left[\left(y-y_{e}\right) v_{y}\left(x, y_{e}\right)+\left(\left(y-y_{e}\right)^{2}-\frac{k_{e}^{2}}{3}\right) \frac{v_{y y}\left(x, y_{e}\right)}{2}\right] d y \\
= & \iint_{e} u_{x x}\left[\left(y-y_{e}\right) v_{y}\left(x, y_{e}\right)+\left(\left(y-y_{e}\right)^{2}-\frac{k_{e}^{2}}{3}\right) \frac{v_{y y}\left(x, y_{e}\right)}{2}\right] d x d y
\end{aligned}
$$

where we have used that

$$
\left(\left(y-y_{e}\right) v_{y}\left(x, y_{e}\right)+\left[\left(y-y_{e}\right)^{2}-\frac{k_{e}^{2}}{3}\right] \frac{v_{y y}\left(x, y_{e}\right)}{2}\right)_{x}=0
$$

based on $v_{x y}=v_{x y y}=0$ for $Q_{1}^{r o t}$ and $E Q_{1}^{\text {rot }}$ elements.

Similarly, we have

$$
\begin{aligned}
& \left(\int_{\ell_{2}}-\int_{\ell_{4}}\right) u_{y}(v-\bar{v}) d x \\
= & \iint_{e} u_{y y}\left[\left(x-x_{e}\right) v_{x}\left(x_{e}, y\right)+\left(\left(x-x_{e}\right)^{2}-\frac{h_{e}^{2}}{3}\right) \frac{v_{x x}\left(x_{e}, y\right)}{2}\right] d x d y .
\end{aligned}
$$

Hence for both $Q_{1}^{\text {rot }}$ and $E Q_{1}^{\text {rot }}$, we obtain from (3.18), (3.22) and (3.24),

$$
a_{h}\left(u-R_{h} u, v\right)=\sum_{e} \iint_{e} u_{x x}\left[\left(y-y_{e}\right) v_{y}\left(x, y_{e}\right)+\left(\left(y-y_{e}\right)^{2}-\frac{k_{e}^{2}}{3}\right) \frac{v_{y y}\left(x, y_{e}\right)}{2}\right]
$$

$$
+\sum_{e} \iint_{e} u_{y y}\left[\left(x-x_{e}\right) v_{x}\left(x_{e}, y\right)+\left(\left(x-x_{e}\right)^{2}-\frac{h_{e}^{2}}{3}\right) \frac{v_{x x}\left(x_{e}, y\right)}{2}\right] .
$$

The desired result (3.2) in Lemma 3.1 follows from Lemma 3.4. This completes the proof of Lemma 3.1 .

3.2. Proof of Lemma 3.2. Denote $B(u, v)=\iint_{\widehat{e}}\left(u-u_{I}\right) v$, where $\widehat{e}=[-1,1]^{2}$ in Figure 2. For $u \in E Q_{1}^{\text {rot }}$, we have $\iint_{\widehat{e}}\left(u-u_{I}\right) v=0$. For $u \in P_{3} \backslash E Q_{1}^{\text {rot }}$, the integration terms needed are given in Table 1. In Table 1 and other tables given below, the zero values can be easily seen by checking odd polynomials with respect 
to $x$ or $y$, and the zero values with "+" in the tables are confirmed by real integral evaluation. Hence, we only examine those zeros with "+" and the nontrivial terms. First, take $u=x^{2} y$ and $v=y$ for example. We have

$$
B\left(x^{2} y, y\right)=\iint_{\widehat{e}}\left(x^{2} y-\frac{y}{3}\right) y=0 .
$$

Similarly, for $u=x y^{2}$ and $v=x$,

$$
B\left(x y^{2}, x\right)=0 \text {. }
$$

Next, we examine the nontrivial terms in Table 1. When $u=x^{3}$ and $v=x$,

$$
B\left(x^{3}, x\right)=\iint_{\widehat{e}}\left(x^{3}-x\right) x=\iint_{\widehat{e}}\left(x^{4}-x^{2}\right)=-\frac{8}{15}=-\frac{1}{45} \iint_{\widehat{e}} u_{x x x} v_{x} .
$$

Similarly, when $u=y^{3}$ and $v=y$,

$$
B\left(y^{3}, y\right)=\iint_{\widehat{e}}\left(y^{3}-y\right) y=\iint_{\widehat{e}}\left(y^{4}-y^{2}\right)=-\frac{8}{15}=-\frac{1}{45} \iint_{\widehat{e}} u_{y y y} v_{y} .
$$

Define the new function

$$
H(u, v)=B(u, v)+\frac{1}{45} \iint_{\widehat{e}} u_{x x x} v_{x}+\frac{1}{45} \iint_{\widehat{e}} u_{y y y} v_{y} .
$$

Hence for $u \in P_{3}, H(u, v)=0$, and then from Lemma 2.2 .

$$
|H(u, v)| \leq C|u|_{4, \widehat{e}}|v|_{1, \widehat{e}} .
$$

Denote $e=\square_{i j}=\left[x_{e}-h_{e}, x_{e}+h_{e}\right] \times\left[y_{e}-k_{e}, y_{e}+k_{e}\right]$ with the boundary lengths $2 h_{e}$ and $2 k_{e}$ (see Figure 2), where $h_{e}=O(h), k_{e}=O(h) \max \left\{\frac{h_{e}}{k_{e}}, \frac{k_{e}}{h_{e}}\right\} \leq C_{0}$, and $C_{0}$ is a constant independent of $h$. Define an affine transformation $T:(x, y) \rightarrow(\widehat{x}, \widehat{y})$ with

$$
\widehat{x}=\frac{x-x_{e}}{h_{e}}, \widehat{y}=\frac{y-y_{e}}{k_{e}} .
$$

Then, under $T$, we have that $e \rightarrow \widehat{e}=[-1,1]^{2}$ and the following equations:

$$
\begin{array}{rlrl}
\widehat{u}(\widehat{x}, \widehat{y}) & =u(x, y), & \widehat{u}_{I}(\widehat{x}, \widehat{y})=u_{I}(x, y), \\
d \widehat{x}=\frac{d x}{h_{e}}, & d \widehat{y}=\frac{d y}{k_{e}}, \\
\widehat{u}_{\widehat{x}}=h_{e} u_{x}, & \widehat{u}_{\widehat{y}}=k_{e} u_{y} .
\end{array}
$$

By the affine transformation $T$ in (3.32) we have

$$
\begin{aligned}
& \iint_{e}\left(u-u_{I}\right) v=h_{e} k_{e} \iint_{\widehat{e}}\left(u-u_{I}\right) v \\
& =h_{e} k_{e}\left[-\frac{1}{45} \iint_{\widehat{e}} u_{x x x} v_{x}-\frac{1}{45} \iint_{\widehat{e}} u_{y y y} v_{y}+O(1)|u|_{4, \widehat{e}}|v|_{1, \widehat{e}}\right] \\
& =-\frac{1}{45}\left[h_{e}^{4} \iint_{e} u_{x x x} v_{x}+k_{e}^{4} \iint_{e} u_{y y y} u_{y}\right]+O\left(h^{5}\right)|u|_{4, e}|v|_{1, e} .
\end{aligned}
$$

This is the desired result (3.4) and completes the proof of Lemma 3.2.

\footnotetext{
${ }^{4}$ For simplicity, we omit the hat notation on the top in the integral of $\widehat{e}$. For instance, the integration $\iint_{\widehat{e}} \widehat{u}_{\widehat{x} \widehat{x} \widehat{x}} \widehat{v}_{\widehat{x}}$ is simplified as $\iint_{\widehat{e}} u_{x x x} v_{x}$ in (3.33).
} 
TABLE 2. The integration, $\iint_{\widehat{e}}\left(u-u_{I}\right) v$ for $u \in P_{3} \backslash Q_{1}^{\text {rot }}, v \in$ $Q_{1}^{\text {rot }}=\operatorname{span}\left\{1, x, y, x^{2}-y^{2}\right\}$, where $\widehat{e}=[-1,1]^{2}$ and the sign $0^{+}$ denotes that the computed integrals are zero.

\begin{tabular}{|c||c|c|c||c|}
\hline$u$ & $x^{2}$ & $x y$ & $y^{2}$ & $x^{3}$ \\
\hline$u_{I}$ & $\frac{2}{3}+\frac{1}{2}\left(x^{2}-y^{2}\right)$ & 0 & $\frac{2}{3}-\frac{1}{2}\left(x^{2}-y^{2}\right)$ & $x$ \\
\hline$u-u_{I}$ & $\frac{1}{2}\left(x^{2}+y^{2}\right)-\frac{2}{3}$ & $x y$ & $\frac{1}{2}\left(x^{2}+y^{2}\right)-\frac{2}{3}$ & $x^{3}-x$ \\
\hline \hline $\iint_{\widehat{e}}\left(u-u_{I}\right)$ & $-\frac{4}{3}$ & 0 & $-\frac{4}{3}$ & 0 \\
\hline $\iint_{\widehat{e}}\left(u-u_{I}\right) x$ & 0 & 0 & 0 & $-\frac{8}{15}$ \\
\hline $\iint_{\widehat{e}}\left(u-u_{I}\right) y$ & 0 & 0 & 0 & 0 \\
\hline $\iint_{\widehat{e}}\left(u-u_{I}\right)\left(x^{2}-y^{2}\right)$ & $0^{+}$ & 0 & $0^{+}$ & 0 \\
\hline$x^{2} y$ & $x y^{2}$ & $y^{3}$ & Notes & \\
\hline$\frac{1}{3} y$ & $\frac{1}{3} x$ & $y$ & $/$ & \\
\hline$x^{2} y-\frac{1}{3} y$ & $x y^{2}-\frac{1}{3} x$ & $y^{3}-y$ & $/$ & \\
\hline \hline 0 & 0 & 0 & $v=1$ \\
\hline 0 & $0^{+}$ & 0 & $v=x$ \\
\hline $0^{+}$ & 0 & $-\frac{8}{15}$ & $v=y$ \\
\hline 0 & 0 & 0 & $v=x^{2}-y^{2}$ & \\
\end{tabular}

3.3. Proof of Lemma 3.3. Denote $B(u, v)=\iint_{\widehat{e}}\left(u-u_{I}\right) v$. For $u \in P_{3} \backslash Q_{1}^{r o t}$, the integration is given in Table 2. Let us check the terms with $0^{+}$and the nontrivial terms in Table 2. First for $u=x^{2}$ and $v=x^{2}-y^{2}$, we have

$$
\begin{aligned}
\iint_{\widehat{e}}\left(u-u_{I}\right) v & =\iint_{\widehat{e}}\left(\frac{1}{2}\left(x^{2}+y^{2}\right)-\frac{2}{3}\right)\left(x^{2}-y^{2}\right) \\
& =\iint_{\widehat{e}}\left[\frac{1}{2}\left(x^{4}-y^{4}\right)-\frac{2}{3}\left(x^{2}-y^{2}\right)\right]=0,
\end{aligned}
$$

where we have used the symmetry: $\iint_{\widehat{e}} x^{2}=\iint_{\widehat{e}} y^{2}$ and $\iint_{\widehat{e}} x^{4}=\iint_{\widehat{e}} y^{4}$. Similarly, for $u=y^{2}$ and $v=x^{2}-y^{2}$,

$$
\iint_{\widehat{e}}\left(u-u_{I}\right) v=0
$$

Next, we examine the nontrivial terms. When $u=x^{2}$ and $v=1$,

$$
\iint_{\widehat{e}}\left(u-u_{I}\right) v=\iint_{\widehat{e}}\left[\frac{1}{2}\left(x^{2}+y^{2}\right)-\frac{2}{3}\right]=-\frac{4}{3}=-\frac{1}{6} \iint_{\widehat{e}} u_{x x} v .
$$

Similarly, when $u=y^{2}$ and $v=1$,

$$
\iint_{\widehat{e}}\left(u-u_{I}\right) v=-\frac{4}{3}=-\frac{1}{6} \iint_{\widehat{e}} u_{y y} v .
$$

Define a functional

$$
H(u, v)=B(u, v)+\frac{1}{6} \iint_{\widehat{e}} u_{x x} v+\frac{1}{6} \iint_{\widehat{e}} u_{y y} v .
$$

Hence for $u \in P_{2}, H(u, v)=0, \forall v \in Q_{1}^{r o t}$, and then from Lemma 2.2.

$$
|H(u, v)| \leq C|u|_{3, \widehat{e}}|v|_{0, \widehat{e}} .
$$


Then we have

$$
B(u, v)=-\frac{1}{6} \iint_{\widehat{e}} u_{x x} v-\frac{1}{6} \iint_{\widehat{e}} u_{y y} v+O(1)|u|_{3, \widehat{e}}|v|_{0, \widehat{e}} .
$$

Below, we consider the additional terms in $P_{3} \backslash P_{2}$, whose results are also listed in Table 2. First, when $u=x^{2} y$ and $v=y$, we have

$$
\iint_{\widehat{e}}\left(u-u_{I}\right) v=\iint_{\widehat{e}}\left(x^{2} y-\frac{1}{3} y\right) y=0,
$$

and when $u=x y^{2}$ and $v=x$,

$$
\iint_{\widehat{e}}\left(u-u_{I}\right) v=\iint_{\widehat{e}}\left(x y^{2}-\frac{1}{3} x\right) x=0 .
$$

Next, when $u=x^{3}$ and $v=x$,

$$
\iint_{\widehat{e}}\left(u-u_{I}\right) v=\iint_{\widehat{e}}\left(x^{3}-x\right) x=\iint_{\widehat{e}}\left(x^{4}-x^{2}\right)=-\frac{8}{15},
$$

and when $u=y^{3}$ and $v=y$, similarly

$$
\iint_{\widehat{e}}\left(u-u_{I}\right) v=-\frac{8}{15}
$$

Now we have to recount $H(u, v)$ for those extra nontrivial terms of $P_{3} \backslash P_{2}$, and obtain from (3.38):

(1) When $u=x^{2} y$ and $v=y$,

$H(u, v)=B\left(x^{2} y, y\right)+\frac{1}{6} \iint_{\widehat{e}} u_{x x} v+\frac{1}{6} \iint_{\widehat{e}} u_{y y} v=0+\frac{4}{9}+0=\frac{4}{9}=\frac{1}{18} \iint_{\widehat{e}} u_{x x y} v_{y}$.

(2) When $u=x y^{2}$ and $v=x$, similarly

$$
H(u, v)=\frac{4}{9}=\frac{1}{18} \iint_{\widehat{e}} u_{x y y} v_{x} .
$$

(3) When $u=x^{3}$ and $v=x$,

$$
\begin{aligned}
H(u, v) & =B\left(x^{3}, x\right)+\frac{1}{6} \iint_{\widehat{e}} u_{x x} v=-\frac{8}{15}+\frac{1}{6} \iint_{\widehat{e}} 6 x^{2} \\
& =-\frac{8}{15}+\frac{4}{3}=\frac{4}{5}=\frac{1}{30} \iint_{\widehat{e}} u_{x x x} v_{x} .
\end{aligned}
$$

(4) When $u=y^{3}$ and $v=y$, similarly

$$
H(u, v)=\frac{4}{5}=\frac{1}{30} \iint_{\widehat{e}} u_{y y y} v_{y} .
$$

Hence we define a new functional

$$
\begin{aligned}
X(u, v)= & H(u, v)-\frac{1}{30} \iint_{\widehat{e}} u_{x x x} v_{x}-\frac{1}{30} \iint_{\widehat{e}} u_{y y y} v_{y} \\
& -\frac{1}{18} \iint_{\widehat{e}} u_{x x y} v_{y}-\frac{1}{18} \iint_{\widehat{e}} u_{x y y} v_{x} .
\end{aligned}
$$

Obviously, for $u \in P_{3}, H(u, v)=0, v \in Q_{1}^{\text {rot }}$, and then from Lemma 2.2.

$$
X(u, v) \leq C|u|_{4, \widehat{e}}|v|_{1, \widehat{e}} .
$$


Then, we conclude that

$$
\begin{aligned}
B(u, v)= & -\frac{1}{6} \iint_{\widehat{e}}\left(u_{x x}+u_{y y}\right) v+\frac{1}{30} \iint_{\widehat{e}}\left(u_{x x x} v_{x}+u_{y y y} v_{y}\right) \\
& +\frac{1}{18} \iint_{\widehat{e}}\left(u_{x x y} v_{y}+u_{x y y} v_{x}\right)+O(1)|u|_{4, \widehat{e}}|v|_{1, \widehat{e}} .
\end{aligned}
$$

The desired result (3.5) follows by the proof techniques via the affine transformation $T$ in (3.32). This completes the proof of Lemma 3.3 .

TABLE 3. The integration, $\iint_{\widehat{e}} u_{x x} D(v)$ for $u \in P_{4} \backslash E Q_{1}^{r o t}, v \in$ $E Q_{1}^{r o t}=\operatorname{span}\left\{1, x, y, x^{2}, y^{2}\right\}$ and $D(v) \in \operatorname{span}\left\{1,0,1,0, y^{2}-\frac{1}{3}\right\}$, where $\widehat{e}=[-1,1]^{2}$ and the sign "/" denotes the zero of integrals due to $u_{x x}=0$.

\begin{tabular}{|c||c|c|c||c|c|c||c|c|c|c|}
\hline$u$ & 1 & $x$ & $y$ & $x^{2}$ & $y^{2}$ & $x y$ & $x^{3}$ & $x^{2} y$ & $x y^{2}$ & $y^{3}$ \\
\hline$u_{x x}$ & 0 & 0 & 0 & 2 & 0 & 0 & $6 x$ & $2 y$ & 0 & 0 \\
\hline $\iint_{\widehat{e}} u_{x x} y$ & $/$ & $/$ & $/$ & 0 & $/$ & $/$ & 0 & $\frac{8}{3}$ & $/$ & $/$ \\
\hline \hline $\iint_{\widehat{e}} u_{x x}\left(y^{2}-\frac{1}{3}\right)$ & $/$ & $/$ & $/$ & $0^{+}$ & $/$ & $/$ & 0 & 0 & $/$ & $/$ \\
\hline
\end{tabular}

\begin{tabular}{|c|c|c|c|c||c|}
\hline$x^{4}$ & $x^{3} y$ & $x y^{3}$ & $y^{4}$ & $x^{2} y^{2}$ & $D(v)$ \\
\hline $12 x^{2}$ & $6 x y$ & 0 & 0 & $2 y^{2}$ & \\
\hline 0 & 0 & $/$ & $/$ & 0 & $v=y, D(v)=y$ \\
\hline \hline 0 & 0 & $/$ & $/$ & $\frac{32}{45}$ & $v=y^{2}, D(v)=y^{2}-\frac{1}{3}$ \\
\hline
\end{tabular}

3.4. Proof of Lemma 3.4. Denote $D(v)=y v_{y}(x, 0)+\left(y^{2}-\frac{1}{3}\right) \frac{v_{y y}(x, 0)}{2}$ on $\hat{e}$ and

$$
B(u, v)=\iint_{\widehat{e}} u_{x x}\left[y v_{y}(x, 0)+\left(y^{2}-\frac{1}{3}\right) \frac{v_{y y}(x, 0)}{2}\right]=\iint_{\widehat{e}} u_{x x} D(v),
$$

where $v \in \operatorname{span}\left\{1, x, y, x^{2}, y^{2}\right\}$ and $D(v)=\operatorname{span}\left\{0,0, y, 0, y^{2}-\frac{1}{3}\right\}$. We list in Table 3 the integration $\iint_{\widehat{e}} u_{x x} D(v)$ for $u \in P_{4}$ and $v \in E Q_{1}^{r o t}$. Let us check the terms with $0^{+}$and the nontrivial terms in Table 3. First, when $u=x^{2}, v=y^{2}$ and $D(v)=y^{2}-\frac{1}{3}$, the integral is zero:

$$
\iint_{\widehat{e}} u_{x x} D(v)=2 \iint_{\widehat{e}}\left(y^{2}-\frac{1}{3}\right)=0 .
$$

Hence for $u \in P_{2}, B(u, v)=0, v \in E Q_{1}^{r o t}$, and then from Lemma 2.2.

$$
|B(u, v)| \leq C|u|_{3, \widehat{e}}|v|_{1, \widehat{e}} .
$$

Next consider $u \in P_{3} / P_{2}$. When $u=x^{2} y$ and $v=y$,

$$
\iint_{\widehat{e}} u_{x x} D(v)=\iint_{\widehat{e}} 2 y^{2}=\frac{8}{3}=\frac{1}{3} \iint_{\widehat{e}} u_{x x y} v_{y} .
$$

Also $B(u, v)=0$ for $u=x^{3}, x y^{2}, y^{3}$ and $v \in E Q_{1}^{\text {rot }}$ (see Table [3). Define a functional

$$
H(u, v)=B(u, v)-\frac{1}{3} \iint_{\widehat{e}} u_{x x y} v_{y} .
$$


For $u \in P_{3}, H(u, v)=0$. From Lemma 2.2

$$
|H(u, v)| \leq C|u|_{4, \widehat{e}}|v|_{1, \widehat{e}},
$$

which yields

$$
B(u, v)=\frac{1}{3} \iint_{\widehat{e}} u_{x x y} v_{y}+O(1)|u|_{4, \widehat{e}}|v|_{1, \widehat{e}} .
$$

By the affine transformation (3.32), we have

$$
\begin{aligned}
& \iint_{e} u_{x x}\left[\left(y-y_{e}\right) v_{y}\left(x, y_{e}\right)+\left(\left(y-y_{e}\right)^{2}-\frac{k_{e}^{2}}{3}\right) \frac{v_{y y}\left(x, y_{e}\right)}{2}\right] \\
& =\frac{k_{e}}{h_{e}} B(\widehat{u}, \widehat{v})=\frac{k_{e}}{h_{e}}\left[\frac{1}{3} \iint_{\widehat{e}} \widehat{u}_{\widehat{x} \widehat{x} \widehat{y}} \widehat{v}_{y}+O(1)|\widehat{u}|_{4, \widehat{e}}|\widehat{v}|_{1, \widehat{e}}\right] \\
& =\frac{k_{e}^{2}}{3} \iint_{e} u_{x x y} v_{y}+O\left(h^{3}\right)|u|_{4, e}|v|_{1, e} .
\end{aligned}
$$

To discover the higher remainders of $O\left(h^{4}\right)$, we should also consider $v \in P_{4} \backslash P_{3}$; the additional integrations are listed in Table 3. Below we consider the nontrivial terms only. For $u=x^{2} y^{2}, v=y^{2}$ and $D(v)=y^{2}-\frac{1}{3}$, we have

$$
\iint_{\widehat{e}} u_{x x} D(v)=\iint_{\widehat{e}} 2 y^{2}\left(y^{2}-\frac{1}{3}\right)=\frac{32}{45},
$$

which gives

$$
\begin{aligned}
H(u, v)=H\left(x^{2} y^{2}, y^{2}\right) & =B\left(x^{2} y^{2}, y^{2}\right)-\frac{1}{3} \iint_{\widehat{e}} u_{x x y} v_{y}=\frac{32}{45}-\frac{1}{3} \iint_{\widehat{e}} 4 y \cdot 2 y \\
& =\frac{32}{45}-\frac{32}{9}=-\frac{128}{45}=-\frac{4}{45} \iint_{\widehat{e}} u_{x x y y} v_{y y} .
\end{aligned}
$$

Now we define a new functional

$$
X(u, v)=H(u, v)+\frac{4}{45} \iint_{\widehat{e}} u_{x x y y} v_{y y} .
$$

Hence for $u \in P_{4}, X(u, v)=0$, and then from Lemma 2.2.

$$
|X(u, v)| \leq C|u|_{5, \hat{e}}|v|_{2, \widehat{e}} .
$$

This yields

$$
B(u, v)=\frac{1}{3} \iint_{\widehat{e}} u_{x x y} v_{y}-\frac{4}{45} \iint_{\widehat{e}} u_{x x y y} v_{y y}+O(1)|u|_{5, \widehat{e}}|v|_{2, \widehat{e}} .
$$

The desired result (3.6) in Lemma 3.4 for $E Q_{1}^{\text {rot }}$ follows from the affine transformation $T$ in (3.32).

Next for $Q_{1}^{\text {rot }}$, we have from Table 3 ,

$$
|B(u, v)| \leq C|u|_{3, \widehat{e}}|v|_{1, \widehat{e}} .
$$

The rest of the proof is exactly the same as that for $E Q_{1}^{r o t}$. This completes the proof of Lemma 3.4 . 


\section{NumERICAL EXPERIMENTS}

In this section, we provide two numerical experiments of the four elements, $Q_{1}, Q_{1}^{r o t}, E Q_{1}^{r o t}$ and Wilson's element for solving (1.1) and (1.2).

4.1. Function $\rho=1$. Consider the eigenvalue problem of Laplace's operator with $\rho=1$,

$$
\begin{aligned}
& -\triangle u=-\left(\frac{\partial^{2} u}{\partial x^{2}}+\frac{\partial^{2} u}{\partial y^{2}}\right)=\lambda u \text { in } S \\
& u=0 \text { on } \Gamma=\partial S
\end{aligned}
$$

where $S=\{(x, y), 0 \leq x, y \leq 1\}$. Then we have the exact eigenfunctions and eigenvalues 5

$$
u_{k, \ell}=2 \sin (k \pi x) \sin (\ell \pi y), \lambda_{k, \ell}=\left(k^{2}+\ell^{2}\right) \pi^{2}, 1 \leq k, \ell \leq N-1 .
$$

Since the minimal and the next minimal eigenvalues, denoted by $\lambda_{1}$ and $\lambda_{2}$, are the most interesting, we only provide their computed results. In Tables 4 and 5 , we list the numerical eigenvalues, their errors and the ratios $=\left|\frac{\varepsilon_{2 h}}{\varepsilon_{h}}\right|$ for all four elements, where $\varepsilon_{h}=\lambda_{h}-\lambda$, and $\lambda_{h}$ and $\lambda$ are the approximate and the true eigenvalues, respectively. Denote $h=1 /(2 N)$ from Figure 2, and $N=2^{m}, m=1,2, \ldots$ When $\left|\frac{\varepsilon_{2 h}}{\varepsilon_{h}}\right| \approx 2^{p}$, we may conclude the empirical convergence rates $O\left(h^{p}\right)$.

For the $Q_{1}$, the $E Q_{1}^{r o t}$ and the Wilson's element, we can see from Tables 4 and 5 that

$$
\lambda_{1, h}-\lambda=O\left(h^{2}\right),
$$

where $\lambda_{\ell, h}$ denotes the computed $\lambda_{\ell}(\ell=1,2)$ at the mesh size $h$. However, for the $Q_{1}^{r o t}$

$$
\begin{aligned}
& \lambda_{1, h}-\lambda_{1}=O\left(h^{4}\right), \\
& \lambda_{2, h}-\lambda_{2}=O\left(h^{2}\right) .
\end{aligned}
$$

Equations (4.2)-(4.4) agree with those in (1.15) perfectly. The high convergence rate $O\left(h^{4}\right)$ in (4.3) results from the symmetry of $u_{x x}=u_{y y}$ for the eigenfunction $u(x, y)$ corresponding to $\lambda_{1}$.

From Table 4, we can find the following relative errors of $\lambda_{1}$ at $N=32$ :

$$
\frac{\lambda_{1, h}-\lambda_{1}}{\lambda_{1}}=0.803(-3),-0.387(-6),-0.802(-3),-0.240(-2),
$$

for $Q_{1}, Q_{1}^{r o t}, E Q_{1}^{r o t}$ and Wilson's elements, respectively. From (4.5) we can see that $Q_{1}$ provides an upper bound due to a positive relative error, and $E Q_{1}^{r o t}$ and Wilson's elements provide lower bounds due to negative relative errors. From Tables 4 and 5. the $Q_{1}^{\text {rot }}$ provide the lower and the upper bounds for $\lambda_{1}$ and $\lambda_{2}$, respectively.

To verify (1.16) we have computed $\widehat{E}_{h}=\left.E_{h}\right|_{Q_{1}^{\text {rot }}}-\frac{1}{2}\left(\left.E_{h}\right|_{Q_{1}}+\left.E_{h}\right|_{E Q_{1}^{\text {rot }}}\right)$, where $E_{h}=\lambda_{1, h}-\lambda_{1}$. Table 6 lists the results to display the $O\left(h^{4}\right)$ convergence rate perfectly.

More importantly, the expansions of eigenvalues can be applied to raise the accuracy by the extrapolation techniques. Based on the computed eigenvalues in

\footnotetext{
${ }^{5}$ The constant 2 of the eigenfunctions in 4.1 is used for $(u, u)=1$.
} 
Tables 4 and 5, we may use the following extrapolation formulas for $\lambda_{1, h}$ :

$$
\lambda_{h}^{(k)}=\frac{2^{2 k} \lambda_{h}^{(k-1)}-\lambda_{2 h}^{(k-1)}}{2^{2 k}-1}, k=1,2,3,4,
$$

for $Q_{1}, E Q_{1}^{\text {rot }}$ and Wilson's elements, where $\lambda_{h}^{0}=\lambda_{h}$. Eq. (4.6) is also used for $\lambda_{2, h}$ by the $Q_{1}^{\text {rot }}$. Since the $\lambda_{1, h}$ by the $Q_{1}^{\text {rot }}$ has the higher convergence rate, the following extrapolation formulas should be used:

$$
\lambda_{h}^{(k)}=\frac{2^{2 k+2} \lambda_{h}^{(k-1)}-\lambda_{2 h}^{(k-1)}}{2^{2 k+2}-1}, k=1,2,3,4 .
$$

Note that in (4.6) and (4.7), $\lambda_{h}^{(1)}$ denotes the first level of extrapolation. In computation, we have computed from the first to the fourth levels of extrapolation. Such a procedure is like that in the Romberg integration. All the extrapolation results are listed in Tables 7 and 8 for $\lambda_{1, h}$ by $Q_{1}^{\text {rot }}$ and $E Q_{1}^{\text {rot }}$. From Tables 7 and 8 we can see

$$
\begin{aligned}
& \lambda_{1, h}^{(1)}-\lambda=O\left(h^{4}\right) \text { for } E Q_{1}^{\text {rot }}, \\
& \lambda_{1, h}^{(1)}-\lambda=O\left(h^{6}\right) \text { for } Q_{1}^{\text {rot }},
\end{aligned}
$$

where $\lambda_{1, h}^{(1)}$ is the better approximation of $\lambda_{1, h}$ at the first level of extrapolation. Below, we list the following eigenvalues at the first and fourth levels of extrapolation:

$$
\begin{aligned}
& \frac{\lambda_{1, h}^{(1)}-\lambda_{1}}{\lambda_{1}}=0.472(-9),-0.512(-5), \\
& \frac{\lambda_{1, h}^{(4)}-\lambda_{1}}{\lambda_{1}}=-0.135(-13),-0.454(-8),
\end{aligned}
$$

for $Q_{1}^{\text {rot }}$ and $E Q_{1}^{\text {rot }}$ at $N=32$ respectively. Evidently, the errors in (4.10) and (4.11) are much smaller than those in (4.5). Interestingly, the $\lambda_{1, h}^{(4)}=19.73920880217845$ by the $Q_{1}^{\text {rot }}$ has 14 significant digits, which is the most accurate value in our computation.

Suppose that we only carry out the computation for $N=2,4,8$, but not for $N=16$ and $N=36$ due to some reasons (e.g., the limitation of computer memory or the CPU time). Based on those results, we may use (4.6) and (4.7) until the second level of extrapolation only. The corresponding results are found from Tables 7 and 8 at $N=8$ :

$$
\left|\frac{\lambda_{1, h}^{(2)}-\lambda_{1}}{\lambda_{1}}\right|=0.422(-6), 0.347(-3),
$$

for $Q_{1}^{\text {rot }}$ and $E Q_{1}^{\text {rot }}$ respectively. The relative errors in (4.12) are close to those in (4.5), but their signs may be changed. This fact displays a significance of the extrapolation, based on the expansions of eigenvalue solutions given in this paper.

The above examination is for the convergence rate; it is crucial to scrutinize numerically the principal terms of the error expansions in (1.15). First, take $E Q_{1}^{\text {rot }}$ for $\lambda_{1}$ for example. Since the corresponding eigenfunction $u_{1,1}=2 \sin (\pi x) \sin (\pi y)$ from (4.1), we have the principal term from (1.15),

$$
E_{1}=-\frac{2 h^{2}}{3} \iint_{S} u_{x y}^{2}=-\frac{2 h^{2} \pi^{4}}{3}=-\frac{\pi^{4}}{6 N^{2}}
$$


where we have used $h=\frac{1}{2 N}$. Then the relative value is given by

$$
\bar{\epsilon}_{1}=\frac{E_{1}}{\lambda_{1}}=-\frac{\pi^{4}}{6 N^{2}\left(2 \pi^{2}\right)}=-\frac{\pi^{2}}{12 N^{2}} .
$$

Based on (4.14), for $N=2,4,8,16,32$, we obtain respectively

$(4.15) \bar{\epsilon}_{1}=-0.206,-0.514(-1),-0.129(-1),-0.321(-2),-0.803(-3)$.

Eq. (4.15) coincides with the numerical data in Table 4 for $E Q_{1}^{r o t}$ very well, which verifies the principal term in (4.13).

Next, consider $Q_{1}^{\text {rot }}$ for $\lambda_{2}$. Since the corresponding eigenfunction $u_{2,1}=$ $2 \sin (2 \pi x) \sin (\pi y)$ from (4.1) with $u_{x x} \neq u_{y y}$, we have the principal term from (1.15)

$$
E_{1}=\frac{h^{2}}{6} \iint_{S}\left(u_{x x}-u_{y y}\right)^{2}=\frac{3 h^{2} \pi^{4}}{2}=\frac{3 \pi^{4}}{8 N^{2}}
$$

which gives

$$
\bar{\epsilon}_{2}=\frac{E_{2}}{\lambda_{2}}=\frac{3 \pi^{4}}{8 N^{2}\left(5 \pi^{2}\right)}=\frac{3 \pi^{2}}{40 N^{2}} .
$$

Based on (4.17), for $N=2,4,8,16,32$, we obtain respectively

$$
\bar{\epsilon}_{2}=0.185,0.463(-1), 0.116(-1), 0.289(-2), 0.723(-3) .
$$

Eq. (4.18) also coincides with the numerical data in Table 5 for $Q_{1}^{\text {rot }}$, which verifies the principal term in (4.16).

4.2. Function $\rho \neq 1$. Since the error analysis is valid for the function $\rho=\rho(x, y) \geq$ $\rho_{0}>0$, to verify the analysis made, we also carry out the numerical experiments for $\rho \neq 1$. Choose

$$
\rho=\rho(x, y)=1+\left(x-\frac{1}{2}\right)\left(y-\frac{1}{2}\right),
$$

which is symmetric with respect to $x$ and $y$. We have

$$
\begin{aligned}
& -\triangle u=-\left(\frac{\partial^{2} u}{\partial x^{2}}+\frac{\partial^{2} u}{\partial y^{2}}\right)=\lambda \rho u \text { in } S, \\
& u=0 \text { on } \Gamma=\partial S
\end{aligned}
$$

where $S$ is also the unit square. For the $\rho$ in (4.19), we may evaluate $\iint_{S} \rho u v$ in (1.5) exactly. The FEM as (1.3) can be easily performed. We provide the results for $\lambda_{1}$ by $Q_{1}^{\text {rot }}$ and $E Q_{1}^{\text {rot }}$ only, and list them in Tables 10 and 11. Since for $\rho$ in (4.19), the true solution of $\lambda_{1}$ is unknown, we may compute the ratios of sequential errors to display the empirical convergence rates 6 The numerical solutions, the sequential errors and their ratios are listed in Tables 10] and 11] for $Q_{1}^{\text {rot }}$ and $E Q_{1}^{\text {rot }}$. Since only the sign of $\varepsilon^{(0)}$ is significant, it is listed in Tables 10 and 11] From Table 11. we can see the sequential errors

\footnotetext{
${ }^{6}$ An a posteriori error may be evaluated as follows. Since $Q_{1}^{\text {rot }}$ may provide the most accurate solution, we may choose $\lambda_{1, h}^{(4)}=19.7322552487$ in Table 10 as the true solution. Then the errors such as those in Tables 10 and 11 can also be computed.
} 


$$
\frac{\lambda_{1,2 h}-\lambda_{1,4 h}}{\lambda_{1, h}-\lambda_{1,2 h}}=O\left(h^{2}\right),
$$

for $E Q_{1}^{\text {rot }}$ elements. However, from Table 10 .

$$
\frac{\lambda_{1,2 h}-\lambda_{1,4 h}}{\lambda_{1, h}-\lambda_{1,2 h}}=O\left(h^{4}\right),
$$

for the $Q_{1}^{\text {rot }}$ element. The empirical convergence rates of $\lambda_{1}$ are exactly the same as those in Section 4.1 for $\rho=1$.

4.3. Numerical conclusions. Based on the numerical results, we may draw a few important conclusions:

(1) The $Q_{1}$ and the $E Q_{1}^{\text {rot }}$ provide the upper and the lower bounds respectively. The $Q_{1}^{\text {rot }}$ provides the lower bound for $\lambda_{2}$ and other $\lambda$ whose corresponding function $u$ satisfies $u_{x x} \neq u_{y y} 7$

(2) For the minimal eigenvalue $\lambda_{\min }=\lambda_{1}$, the corresponding eigenfunctions satisfy $u_{x x}=u_{y y}$, and the $Q_{1}^{r o t}$ element yields the high $O\left(h^{4}\right)$ convergence rates. Such an ultraconvergence of $Q_{1}^{\text {rot }}$ holds for any eigenvalues whose eigenfunctions are symmetric with respect to $x$ and $y$.

(3) We list in Table 6 the computed results, to show the validation of (1.16).

(4) By the first level of extrapolation, the superconvergence $O\left(h^{4}\right)$ can be obtained by all four FEMs.

(5) For $Q_{1}^{\text {rot }}$, the ultraconvergence for $\lambda_{1}$ as

$$
\lambda_{1, h}^{(i)}-\lambda_{1}=O\left(h^{2 i+4}\right), i=0,1,2,3,
$$

can be achieved numerically by multiple levels of extrapolation; see Table 8

(6) The principal terms of the eigenvalue errors for $Q_{1}^{\text {rot }}$ and $E Q_{1}^{\text {rot }}$ have been verified by our numerical experiments.

Concluding remarks. The new expansions of numerical eigenvalues by four FEMs are summarized in (1.15), whose proof for the two nonconforming elements $Q_{1}^{\text {rot }}$ and $E Q_{1}^{\text {rot }}$ is provided in this paper. Not only can (1.15) display an upper or a lower bound of the FEM solution of leading eigenvalues, but it can also lead to higher superconvergence rates by the extrapolation techniques. All the theoretical analyses have been verified by the numerical experiments in Section 4. Moreover, the best convergence rates have been obtained numerically by multiple levels of extrapolation for both $Q_{1}^{\text {rot }}$ and $E Q_{1}^{\text {rot }}$ elements.

\footnotetext{
${ }^{7}$ Numerically, the $Q_{1}^{\text {rot }}$ also provides the lower bound of $\lambda_{1}$, based on Table 4 for $\rho=1$, and on Table 10 for $\rho \neq 1$.
} 
TABLE 4 . The first eigenvalue solutions $\lambda_{1, h}$ for $-\Delta u=\lambda u$ by the four FEMs, where the true $\lambda_{1}=2 \pi^{2} \stackrel{\doteq}{=} 19.73920880217872$, $\varepsilon_{h}=\frac{\lambda_{1, h}-\lambda_{1}}{\lambda_{1}}$, Ratio $=\left|\frac{\varepsilon_{2 h}}{\varepsilon_{h}}\right|$ and $h=\frac{1}{2 N}$.

\begin{tabular}{|l|c|c|c|c|c|}
\hline$N$ & 2 & 4 & 8 & 16 & 32 \\
\hline$\lambda_{1, h}$ by $Q_{1}$ & 24.000000 & 20.773284 & 19.994161 & 19.802707 & 19.755068 \\
\hline$\lambda_{1, h}$ by $Q_{1}^{\text {rot }}$ & 19.200000 & 19.707183 & 19.737241 & 19.739086 & 19.739201 \\
\hline$\lambda_{1, h}$ by $E Q_{1}^{\text {rot }}$ & 16.848019 & 18.818638 & 19.491886 & 19.676196 & 19.723380 \\
\hline$\lambda_{1, h}$ by Wilson's & 13.321013 & 17.296011 & 19.023223 & 19.551919 & 19.691833 \\
\hline \hline$\varepsilon_{h}$ by $Q_{1}$ & 0.216 & $0.524(-1)$ & $0.129(-1)$ & $0.322(-2)$ & $0.803(-3)$ \\
\hline$\varepsilon_{h}$ by $Q_{1}^{\text {rot }}$ & $-0.273(-1)$ & $-0.162(-2)$ & $-0.997(-4)$ & $-0.620(-5)$ & $-0.387(-6)$ \\
\hline$\varepsilon_{h}$ by $E Q_{1}^{\text {rot }}$ & -0.146 & $-0.466(-1)$ & $-0.125(-1)$ & $-0.319(-2)$ & $-0.802(-3)$ \\
\hline$\varepsilon_{h}$ by Wilson's & -0.325 & -0.124 & $-0.363(-1)$ & $-0.949(-2)$ & $-0.240(-2)$ \\
\hline \hline Ratio by $Q_{1}$ & $/$ & 4.12 & 4.06 & 4.02 & 4.00 \\
\hline Ratio by $Q_{1}^{\text {rot }}$ & $/$ & 16.8 & 16.3 & 16.1 & 16.0 \\
\hline Ratio by $E Q_{1}^{\text {rot }}$ & $/$ & 3.14 & 3.72 & 3.92 & 3.98 \\
\hline Ratio by Wilson's & $/$ & 2.63 & 3.41 & 3.82 & 3.95 \\
\hline
\end{tabular}

TABLE 5. The second eigenvalue solutions $\lambda_{2, h}$ for $-\Delta u=\lambda u$ by the four FEMs, where the true $\lambda_{2}=5 \pi^{2} \doteq 49.3480220054$, $\varepsilon_{h}=\frac{\lambda_{2, h}-\lambda_{2}}{\lambda_{2}}$, Ratio $=\left|\frac{\varepsilon_{2 h}}{\varepsilon_{h}}\right|$ and $h=\frac{1}{2 N}$.

\begin{tabular}{|l|c|c|c|c|c|}
\hline$N$ & 2 & 4 & 8 & 16 & 32 \\
\hline$\lambda_{2, h}$ by $Q_{1}$ & $/$ & 58.3866 & 51.5436 & 49.8897 & 49.4829 \\
\hline$\lambda_{2, h}$ by $Q_{1}^{\text {rot }}$ & 58.5366 & 51.3290 & 49.9022 & 49.4897 & 49.3836 \\
\hline$\lambda_{2, h}$ by $E Q_{1}^{\text {rot }}$ & 41.6696 & 46.3304 & 48.4088 & 49.0993 & \\
\hline$\lambda_{2, h}$ by Wilson's & 21.8182 & 38.6181 & 45.7010 & 48.3394 & \\
\hline \hline$\varepsilon_{h}$ by $Q_{1}$ & $/$ & 0.183 & $0.445(-1)$ & $0.110(-1)$ & $0.273(-2)$ \\
\hline$\varepsilon_{h}$ by $Q_{1}^{\text {rot }}$ & 0.186 & $0.401(-1)$ & $0.112(-1)$ & $0.287(-2)$ & $0.722(-3)$ \\
\hline$\varepsilon_{h}$ by $E Q_{1}^{\text {rot }}$ & -0.156 & $-0.611(-1)$ & $-0.190(-1)$ & $-0.504(-2)$ & \\
\hline$\varepsilon_{h}$ by Wilson's & -0.558 & -0.217 & $-0.739(-1)$ & $-0.204(-1)$ & \\
\hline \hline Ratio by $Q_{1}$ & $/$ & $/$ & 4.12 & 4.05 & 4.01 \\
\hline Ratio by $Q_{1}^{\text {rot }}$ & $/$ & 4.64 & 3.57 & 3.91 & 3.98 \\
\hline Ratio by $E Q_{1}^{\text {rot }}$ & $/$ & 2.55 & 3.21 & 3.78 & \\
\hline Ratio by Wilson's & $/$ & 2.57 & 2.94 & 3.62 & \\
\hline
\end{tabular}

TABLE 6. The errors $\lambda_{1, h}-\lambda_{1}$ for $Q_{1}, Q_{1}^{\text {rot }}$ and $E Q_{1}^{\text {rot }}$, where $E=\lambda_{1, h}-\lambda_{1}, \widehat{E}_{h}=\left.E_{h}\right|_{Q_{1}^{\text {rot }}}-\frac{1}{2}\left(\left.E_{h}\right|_{Q_{1}}+\left.E_{h}\right|_{E Q_{1}^{\text {rot }}}\right)$, Ratio $=$ $\widehat{E}_{2 h} / \widehat{E}_{h}$ and $h=\frac{1}{2 N}$.

\begin{tabular}{|c|c|c|c|c|c|}
\hline$N$ & 2 & 4 & 8 & 16 & 32 \\
\hline$\lambda_{1, h}$ by $Q_{1}$ & 24.0000000000 & 20.7732840104 & 19.9941613125 & 19.8027073568 & 19.7550682351 \\
\hline$\lambda_{1, h}$ by $Q_{1}^{\text {rot }}$ & 19.2000000000 & 19.7071826998 & 19.7372410126 & 19.7390863687 & 19.7392011588 \\
\hline$\lambda_{1, h}$ by $E Q_{1}^{\text {rot }}$ & 16.8480192154 & 18.8186378768 & 19.4918862529 & 19.6761961558 & 19.7233798827 \\
\hline \hline$E_{h}$ by $Q_{1}$ & 4.26079 & 1.03408 & 0.254953 & $0.634986(-1)$ & $0.158594(-1)$ \\
\hline$E_{h}$ by $Q_{1}^{\text {rot }}$ & -0.539209 & $-0.320261(-1)$ & $-0.196779(-2)$ & $-0.122433(-3)$ & $-0.764336(-5)$ \\
\hline$E_{h}$ by $E Q_{1}^{\text {rot }}$ & -2.89119 & -0.920571 & -0.247323 & $-0.630126(-1)$ & $-0.158289(-1)$ \\
\hline$\widehat{E}_{h}$ & -1.22401 & $-0.887782(-1)$ & $-0.578277(-2)$ & $-0.365388(-3)$ & $-0.229001(-4)$ \\
\hline \hline Ratio & $/$ & 13.79 & 15.35 & 15.83 & 15.96 \\
\hline
\end{tabular}


TABLE 7 . The first $\lambda_{1, h}$ by extrapolation from the $Q_{1}^{\text {rot }}$ solutions, where the true $\lambda_{1}=2 \pi^{2} \doteq 19.73920880217872, \lambda_{1, h}^{(k)}=$ $\frac{2^{2 k+2} \lambda_{1, h}^{(k-1)}-\lambda_{1,2 h}^{(k-1)}}{2^{2 k+2}-1}, \varepsilon_{h}^{(k)}=\frac{\lambda_{1, h}^{(k)}-\lambda_{1}}{\lambda_{1}}, \operatorname{Ratio}(k)=\left|\varepsilon_{2 h}^{(k)} / \varepsilon_{h}^{(k)}\right|, \lambda_{1, h}^{(0)}=$ $\lambda_{1, h}$ and $h=\frac{1}{2 N}$.

\begin{tabular}{|c|c|c|c|c|c|}
\hline$N$ & 2 & 4 & 8 & 16 & 32 \\
\hline$\lambda_{1, h}$ & 19.20000000000000 & 19.70718269984467 & 19.73724101257975 & 19.73908636870386 & 19.73920115882359 \\
\hline$\lambda_{1, h}^{(1)}$ & $/$ & 19.74099487983432 & 19.73924490009542 & 19.73920939244547 & 19.73920881149824 \\
\hline$\lambda_{1, h}^{(2)}$ & $/$ & $/$ & 19.73921712263925 & 19.73920882883198 & 19.73920880227686 \\
\hline$\lambda_{1, h}^{(3)}$ & $/$ & $/$ & $/$ & 19.73920879630725 & 19.73920880217272 \\
\hline$\lambda_{1, h}^{(4)}$ & $/$ & $/$ & $/$ & $/$ & 19.73920880217845 \\
\hline \hline$\varepsilon_{h}^{(0)}$ & $-0.273(-1)$ & $-0.162(-2)$ & $-0.997(-4)$ & $-0.620(-5)$ & $-0.387(-6)$ \\
\hline$\varepsilon_{h}^{(1)}$ & $/$ & $0.905(-4)$ & $0.183(-5)$ & $0.299(-7)$ & $0.472(-9)$ \\
\hline$\varepsilon_{h}^{(2)}$ & $/$ & $/$ & $0.422(-6)$ & $0.135(-8)$ & $0.497(-11)$ \\
\hline$\varepsilon_{h}^{(3)}$ & $/$ & $/$ & $/$ & $-0.297(-9)$ & $-0.304(-12)$ \\
\hline$\varepsilon_{h}^{(4)}$ & $/$ & $/$ & $/$ & $/$ & $-0.135(-13)$ \\
\hline \hline Ratio(0) & $/$ & 16.8 & 16.3 & 16.1 & 16.0 \\
\hline Ratio(1) & $/$ & $/$ & 49.5 & 61.2 & 63.3 \\
\hline Ratio(2) & $/$ & $/$ & $/$ & 312 & 272 \\
\hline Ratio(3) & $/$ & $/$ & & $/$ & 979 \\
\hline
\end{tabular}

TABLE 8 . The first $\lambda_{1, h}$ by extrapolation from the $E Q_{1}^{\text {rot }}$ solutions, where the true $\lambda_{1}=2 \pi^{2} \doteq 19.73920880217872$, where $\lambda_{1, h}^{(k)}=$ $\frac{2^{2 k} \lambda_{1, h}^{(k-1)}-\lambda_{1,2 h}^{(k-1)}}{2^{2 k}-1}, \varepsilon_{h}^{(k)}=\frac{\lambda_{1, h}^{(k)}-\lambda_{1}}{\lambda_{1}}, \operatorname{Ratio}(k)=\left|\varepsilon_{2 h}^{(k)} / \varepsilon_{h}^{(k)}\right|, \lambda_{1, h}^{(0)}=\lambda_{1, h}$ and $h=\frac{1}{2 N}$.

\begin{tabular}{|c|c|c|c|c|c|}
\hline$N$ & 2 & 4 & 8 & 16 & 32 \\
\hline$\lambda_{1, h}$ & 16.8480192154 & 18.8186378768 & 19.4918862529 & 19.6761961558 & 19.7233798827 \\
\hline$\lambda_{1, h}^{(1)}$ & $/$ & 19.4755107640 & 19.7163023782 & 19.7376327901 & 19.7391077917 \\
\hline$\lambda_{1, h}^{(2)}$ & $/$ & $/$ & 19.7323551525 & 19.7390548176 & 19.7392061251 \\
\hline$\lambda_{1, h}^{(3)}$ & $/$ & $/$ & $/$ & 19.7391611615 & 19.7392085268 \\
\hline$\lambda_{1, h}^{(4)}$ & $/$ & $/$ & $/$ & $/$ & 19.7392087126 \\
\hline \hline$\varepsilon_{h}^{(0)}$ & -0.146 & $-0.466(-1)$ & $-0.125(-1)$ & $-0.319(-2)$ & $-0.802(-3)$ \\
\hline$\varepsilon_{h}^{(1)}$ & $/$ & $-0.134(-1)$ & $-0.116(-2)$ & $-0.798(-4)$ & $-0.512(-5)$ \\
\hline$\varepsilon_{h}^{(2)}$ & $/$ & $/$ & $-0.347(-3)$ & $-0.780(-5)$ & $-0.136(-6)$ \\
\hline$\varepsilon_{h}^{(3)}$ & $/$ & $/$ & $/$ & $-0.241(-5)$ & $-0.140(-7)$ \\
\hline$\varepsilon_{h}^{(4)}$ & $/$ & $/$ & $/$ & $/$ & $-0.454(-8)$ \\
\hline \hline Ratio(0) & $/$ & 3.14 & 3.72 & 3.92 & 3.98 \\
\hline Ratio(1) & $/$ & $/$ & 11.5 & 14.5 & 15.6 \\
\hline Ratio(2) & $/$ & $/$ & $/$ & 44.5 & 57.5 \\
\hline Ratio(3) & $/$ & $/$ & $/$ & $/$ & 173 \\
\hline
\end{tabular}


TABLE 9 . The second $\lambda_{2, h}$ by extrapolation from the $Q_{1}^{\text {rot }}$ solutions, where the true $\lambda_{2}=5 \pi^{2} \doteq 49.34802200544679, \lambda_{2, h}^{(k)}=$ $\frac{2^{2 k} \lambda_{2, h}^{(k-1)}-\lambda_{2,2 h}^{(k-1)}}{2^{2 k}-1}, \varepsilon_{h}^{(k)}=\frac{\lambda_{2, h}^{(k)}-\lambda_{2}}{\lambda_{2}}, \operatorname{Ratio}(k)=\left|\varepsilon_{2 h}^{(k)} / \varepsilon_{h}^{(k)}\right|, \lambda_{2, h}^{(0)}=\lambda_{2, h}$ and $h=\frac{1}{2 N}$.

\begin{tabular}{|c|c|c|c|c|c|}
\hline$N$ & 2 & 4 & 8 & 16 & 32 \\
\hline$\lambda_{2, h}$ & 58.5365853659 & 51.3289965050 & 49.9021574073 & 49.4897065300 & 49.3836320249 \\
\hline$\lambda_{2, h}^{(1)}$ & $/$ & 48.9264668847 & 49.4265443748 & 49.3522229042 & 49.3482738566 \\
\hline$\lambda_{2, h}^{(2)}$ & $/$ & $/$ & 49.4598828741 & 49.3472681395 & 49.3480105867 \\
\hline$\lambda_{2, h}^{(3)}$ & $/$ & $/$ & $/$ & 49.3454806040 & 49.3480223716 \\
\hline$\lambda_{2, h}^{(4)}$ & $/$ & $/$ & $/$ & $/$ & 49.3480323393 \\
\hline \hline$\varepsilon_{h}^{(0)}$ & 0.186 & $0.401(-1)$ & $0.112(-1)$ & $0.287(-2)$ & $0.722(-3)$ \\
\hline$\varepsilon_{h}^{(1)}$ & $/$ & $-0.854(-2)$ & $0.159(-2)$ & $0.851(-4)$ & $0.510(-5)$ \\
\hline$\varepsilon_{h}^{(2)}$ & $/$ & $/$ & $0.227(-2)$ & $-0.153(-4)$ & $-0.231(-6)$ \\
\hline$\varepsilon_{h}^{(3)}$ & $/$ & $/$ & $/$ & $-0.515(-4)$ & $0.742(-8)$ \\
\hline$\varepsilon_{h}^{(4)}$ & $/$ & $/$ & $/$ & $/$ & $0.209(-6)$ \\
\hline \hline Ratio(0) & $/$ & 4.64 & 3.57 & 3.91 & 3.98 \\
\hline Ratio(1) & $/$ & $/$ & 5.37 & 18.7 & 16.7 \\
\hline Ratio(2) & $/$ & $/$ & $/$ & 148 & 66.0 \\
\hline Ratio(3) & $/$ & $/$ & $/$ & $/$ & 6940 \\
\hline
\end{tabular}

TABLE 10. The first $\lambda_{1, h}$ for $-\Delta u=\lambda \rho u$ by $Q_{1}^{\text {rot }}$, where $\lambda_{1, h}^{(k)}=$ $\frac{2^{2 k+2} \lambda_{1, h}^{(k-1)}-\lambda_{1,2 h}^{(k-1)}}{2^{2 k+2}-1}, \varepsilon_{h}^{(k)}=\lambda_{1, h}^{(k)}-\lambda_{1,2 h}^{(k)}, \operatorname{Ratio}(k)=\left|\varepsilon_{2 h}^{(k)} / \varepsilon_{h}^{(k)}\right|, \lambda_{1, h}^{(0)}=$ $\lambda_{1, h}$ and $h=\frac{1}{2 N}$.

\begin{tabular}{|c|c|c|c|c|c|}
\hline$N$ & 2 & 4 & 8 & 16 & 32 \\
\hline$\lambda_{1, h}$ & 19.2000000000 & 19.7011813280 & 19.7303925515 & 19.7321409854 & 19.7322481452 \\
\hline$\lambda_{1, h}^{(1)}$ & $/$ & 19.7345933897 & 19.7323399681 & 19.7322575477 & 19.7322552892 \\
\hline$\lambda_{1, h}^{(2)}$ & $/$ & $/$ & 19.7323041995 & 19.7322562394 & 19.7322552534 \\
\hline$\lambda_{1, h}^{(3)}$ & $/$ & $/$ & $/$ & 19.7322560513 & 19.7322552495 \\
\hline$\lambda_{1, h}^{(4)}$ & $/$ & $/$ & $/$ & $/$ & 19.7322552487 \\
\hline \hline$\varepsilon_{h}^{(0)}$ & $/$ & -0.501 & $-0.292(-1)$ & $-0.175(-2)$ & $-0.107(-3)$ \\
\hline$\left|\varepsilon_{h}^{(1)}\right|$ & $/$ & $/$ & $0.225(-2)$ & $0.824(-4)$ & $0.226(-5)$ \\
\hline$\left|\varepsilon_{h}^{(2)}\right|$ & $/$ & $/$ & $/$ & $0.480(-4)$ & $0.986(-6)$ \\
\hline$\left|\varepsilon_{h}^{(3)}\right|$ & $/$ & $/$ & $/$ & $/$ & $0.802(-6)$ \\
\hline \hline Ratio(0) & $/$ & $/$ & 17.16 & 16.71 & 16.32 \\
\hline Ratio(1) & $/$ & $/$ & $/$ & 27.34 & 36.49 \\
\hline Ratio(2) & $/$ & $/$ & $/$ & $/$ & 48.64 \\
\hline
\end{tabular}


TABLE 11. The first $\lambda_{1, h}$ for $-\Delta u=\lambda \rho u$ by $E Q_{1}^{\text {rot }}$, where $\lambda_{1, h}^{(k)}=$ $\frac{2^{2 k} \lambda_{1, h}^{(k-1)}-\lambda_{1,2 h}^{(k-1)}}{2^{2 k}-1}, \varepsilon_{h}^{(k)}=\lambda_{1, h}^{(k)}-\lambda_{1,2 h}^{(k)}, \operatorname{Ratio}(k)=\left|\varepsilon_{2 h}^{(k)} / \varepsilon_{h}^{(k)}\right|, \lambda_{1, h}^{(0)}=$ $\lambda_{1, h}$ and $h=\frac{1}{2 N}$.

\begin{tabular}{|c|c|c|c|c|c|}
\hline$N$ & 2 & 4 & 8 & 16 & 32 \\
\hline$\lambda_{1, h}$ & 17.7264222820 & 19.1414166129 & 19.5800759724 & 19.6984491727 & 19.7286510415 \\
\hline$\lambda_{1, h}^{(1)}$ & $/$ & 19.6130813898 & 19.7262957589 & 19.7379069061 & 19.7387183311 \\
\hline$\lambda_{1, h}^{(2)}$ & $/$ & $/$ & 19.7338433835 & 19.7386809826 & 19.7387724261 \\
\hline$\lambda_{1, h}^{(3)}$ & $/$ & $/$ & $/$ & 19.7387577699 & 19.7387738776 \\
\hline$\lambda_{1, h}^{(4)}$ & $/$ & $/$ & $/$ & $/$ & 19.7387739407 \\
\hline \hline$\varepsilon_{h}^{(0)}$ & $/$ & -1.415 & -0.439 & -0.118 & $-0.302(-1)$ \\
\hline$\left|\varepsilon_{h}^{(1)}\right|$ & $/$ & $/$ & 0.113 & $0.116(-1)$ & $0.811(-3)$ \\
\hline$\left|\varepsilon_{h}^{(2)}\right|$ & $/$ & $/$ & $/$ & $0.484(-2)$ & $0.914(-4)$ \\
\hline$\left|\varepsilon_{h}^{(3)}\right|$ & $/$ & $/$ & $/$ & $/$ & $0.161(-4)$ \\
\hline \hline Ratio(0) & $/$ & $/$ & 3.23 & 3.71 & 3.92 \\
\hline Ratio(1) & $/$ & $/$ & $/$ & 9.75 & 14.31 \\
\hline Ratio(2) & $/$ & $/$ & $/$ & $/$ & 52.90 \\
\hline
\end{tabular}

\section{ACKNOWLEDGEMENTS}

The authors express their sincere thanks to the reviewers for their careful reading and very valuable suggestions. Also, we are indebted to H. J. Shen for the numerical results in Section 4.

\section{REFERENCES}

[1] I. Babuska and J.E. Osborn, Estimates for the errors in eigenvalue and eigenvector approximation by Galerkin methods with particular attention to the case of multiple eigenvalues, SIAM J. Numer. Anal., Vol.24, pp.1249-1276, 1987. MR917451 (88j:65234)

[2] I. Babuska and J.E. Osborn, Finite element-Galerkin approximation of the eigenvalues and eigenvectors of selfadjoint problems, Math. Comp., Vol.52, pp.275-297, 1989. MR962210 (89k:65132)

[3] I. Babuska and J. Osborn, Eigenvalue problems in Finite Element Method (Part I), edited by P.G. Ciarlet and J.L. Lions, pp.641-787, North-Holland, Ansterdam, 1991. MR1115240

[4] H. Blum, Q. Lin and R. Rannacher, Asymptotic error expansion and Richardson extrapolation for linear finite elements, Numer. Math., Vol.49, pp. 11-37, 1986. MR847015 (87m:65172)

[5] J.H. Bramble and S. R. Hilbert, Estimation of linear functionals on Sobolev spaces with application to Fourier transforms and spline interpolation, SIAM J. Numer. Anal., Vol.7, pp. 113-124, 1970. MR0263214(41:7819)

[6] F. Chatelin, Convergence of approximation methods to compute eigenelements of linear operations, SIAM J. Numer. Anal., Vol.10, pp.939-948, 1973. MR0349004 (50:1498)

[7] H. Chen and B. Li, Superconvergence analysis and error expansion for the Wilson nonconforming finite element, Numer. Math., Vol.69, pp.125-140, 1994. MR.1310313 (95k:65105)

[8] G. E. Forsythe, Asymptotic lower bounds for frequencies of certain polygonal membrances, Pacific J. Math., Vol 4, pp.467-480, 1954. MR0063784 (16:179g)

[9] J. Hu, P.-B. Ming and Z.-C. Shi, Nonconforming quadrilateral rotated $Q_{1}$ element for Reissner-Mindlin plate, J. Comp. Math., Vol.21, No.1, pp.25-32, 2003. MR.1974269 (2004c:65143)

[10] W.G. Koluta, Approximation in variationally posed eigenvalue problems, Numer. Math., Vol.29, pp.159-171, 1978. MR 482047(80a:49077)

[11] Z. C. Li, Combined Methods for Elliptic Equations with Singularities, Interfaces and Infinities, Kluwer Academic Publishers, 1998. MR1639538 (99i:65001) 
[12] Q. Lin, Fourth order eigenvalue approximation by extrapolation on domains with reentrant corners, Numer. Math., Vol. 58, pp.631-640,1991. MR.1083525 (92d:65198)

[13] Q. Lin and J. Lin, Finite Element Methods; Accuracy and Improvement, Science Press, Beijing, 2006.

[14] Q. Lin and Q. Zhu, Processing and Post processing for the Finite Element Method (in Chinese), Shanghai Scientific \& Technical Press., 1994.

[15] T. Lü, C.B. Liem and T.M.Shih, The Splitting Extrapolation and Combination Techniques New Techniques of Parallel Solutions for Multi-dimensional Problems (in Chinese), Scientific Publishers, Beijing, 1998.

[16] P. Lua and Q. Lin, High accuracy analysis of the Wilson element, J. Comp. Math., Vol.17, No.2, pp.113-124, 1999. MR.1688000(2000b:65220)

[17] B. Mercier, J. Osborn, J. Rappaz and P.-A. Raviat, Eigenvalue approximation by mixed and hybrid methods, Math. Comp., Vol.36, No.154, pp.427-453, 1981. MR606505 (82b:65108)

[18] J.G. Pierce and R.S. Varga, High order convergence results for the Rayleigh-Ritz method applied to eigenvalue problems: 2. Improved error bounds for eigenfunctions, Numer. Math., Vol.19, pp.155-169, 1972. MR0323133 (48:1491)

[19] R. Rannacher, Nonconforming finite element methods for eigenvalue problems in linear plate theory, Numer. Math., Vol.33, pp.23-43, 1979. MR545740 (80i:65124)

[20] G. Strang and G. Fix, An Analysis of the Finite Element Method, Prentice-Hall Series in Automatic Comp., Prentice-Hall, Englewood Cliffs, NJ, 1973. MR0443377 (56:1747)

[21] H. F. Weinberger, Variational Methods for Eigenvalue Approximation, SIAM, Philadelphia, 1974. MR0400004 (53:3842)

[22] D.-S. Wu, Convergence and superconvergence of Hermite bicubic element for eigenvalue problem of the biharmonic equation, J. Comp. Math., Vol.19, No.2, pp.139-142, 2001. MR1816677 (2001m:65161)

[23] Y.D. Yang, Computable error bounds for an eigenvalue problem in the finite element method, Chinese J. Numer. Math. Appl., Vol.17, No.1, pp.68-77, 1995. MR.1392853(97d:65058)

[24] Y. D. Yang, A posteriori error estimates in Adini finite element for eigenvalue problems, J. Comp. Math., Vol.18, pp.413-418, 2000. MR1773912 (2001c:65140)

Institute of Computational Mathematics and Scientific/Engineering Computing, Academy of Mathematics and System Sciences, Chinese Academy of Sciences, P.O. Box 2719, BeiJing 1000080, China

E-mail address: qlin@lsec.cc.ac.cn

Department of Applied Mathematics, I-Shou University, Taiwan 840

E-mail address: huanght@isu.edu.tw

(Corresponding author) Department of Applied Mathematics, and Department of Computer Science and Engineering, National Sun Yat-Sen University, Kaohsiung, Taiwan 80424

E-mail address: zcli@math.nsysu.edu.tw 